\title{
Single and Composite Hot Subdwarf Stars in the Light of 2MASS Photometry
}

\author{
M. A. Stark and Richard A. Wade \\ Department of Astronomy and Astrophysics, The Pennsylvania State University, University Park, \\ $P A 16802$ \\ stark@astro.psu.edu, wade@astro.psu.edu
}

\begin{abstract}
Utilizing the Two Micron All Sky Survey (2MASS) Second Incremental Data Release Catalog, we have retrieved near-IR magnitudes for several hundred hot subdwarfs (sdO and sdB stars) drawn from the Catalogue of Spectroscopically Identified Hot Subdwarfs (Kilkenny, Heber, \& Drilling 1988, 1992). This sample size greatly exceeds that of previous studies of hot subdwarfs. Examining 2MASS photometry alone or in combination with visual photometry (Johnson $B V$ or Strömgren $u v b y$ ) available in the literature, we show that it is possible to identify hot subdwarf stars that exhibit atypically red IR colors that can be attributed to the presence of an unresolved late type companion. Utilizing this large sample, we attempt for the first time to define an approximately volume limited sample of hot subdwarfs. We discuss the considerations, biases, and difficulties in defining such a sample.

We find that, of the hot subdwarfs in Kilkenny et al., about $40 \%$ in a magnitude limited sample have colors that are consistent with the presence of an unresolved late type companion. Binary stars are over-represented in a magnitude limited sample. In an approximately volume limited sample the fraction of composite-color binaries is about $30 \%$.
\end{abstract}

Subject headings: binaries: general — stars: early-type — stars: horizontal-branch — subdwarfs — infrared: stars

\section{Introduction}

Hot subdwarf stars as a class have effective temperatures exceeding $\sim 20,000 \mathrm{~K}$, with surface gravities higher and luminosities lower than main sequence stars of the same temperature. Spectroscopically, hot subdwarfs are divided into two major subtypes: subdwarf B (sdB) and subdwarf O (sdO). SdB stars have hydrogen-dominated atmospheres with $T_{\text {eff }} \approx 24,000-30,000 \mathrm{~K}$ and exhibit a relatively small dispersion in luminosity (Heber 1986; Saffer et al. 1994; de Boer et al. 1997). They often exhibit weak lines of Helium, Carbon, and other atomic species in comparison with Population I main sequence stars. SdO stars have helium-enriched atmospheres with $T_{\text {eff }}>30,000 \mathrm{~K}$ and 
exhibit a wider range of $\log g$ and luminosity than the sdB stars (Heber 1986; Saffer et al. 1994; de Boer et al. 1997). Hot subdwarf stars (and sdB stars in particular) are the primary source of the "UV upturn" (or "UVX") seen in elliptical galaxies and spiral galaxy bulges (e.g., Greggio \& Renzini 1990; Brown et al. 1997, 2000; O'Connell 1999).

Based on their temperatures and magnitudes, Humason \& Zwicky (1947) proposed that the sdB stars are the field equivalent of Baade's (1944) population II B stars (now known as "extended horizontal branch ${ }^{1}$," or "EHB," stars) which are seen in some globular cluster HR diagrams at the blue end of the horizontal branch. As such, sdB stars in the field are understood to be core helium burning objects with very low hydrogen envelope masses $\left(\mathrm{M}_{\text {env }} \lesssim 0.02 \mathrm{M}_{\odot}\right)$ and total masses of $\mathrm{M} \approx 0.5-0.55 \mathrm{M}_{\odot}$ (Caloi 1972; Saffer et al. 1994). Some sdO stars have colors and luminosities that are consistent with the hottest part of the EHB (least massive $\mathrm{H}$ envelopes). However, there are a number of potential evolutionary paths, e.g. post-AGB evolution, that lead through the rather broad region of the HR diagram occupied by sdO stars. All hot subdwarfs, regardless of their prior evolution, are thought to be direct progenitors of white dwarfs, although they constitute only a small fraction of all stars that evolve to become white dwarfs.

While the current evolutionary status of $\mathrm{sdB}$ stars is relatively well understood, how they actually arrived at the EHB with such low envelope masses is not understood. The spectroscopically inferred masses of field $\mathrm{sdB}$ stars have a very small dispersion. If they evolved directly from red giant progenitors, then their initial masses are constrained to be $\lesssim 2 \mathrm{M}_{\odot}$, leading to a requirement that they lose up to $\sim 1.5 \mathrm{M}_{\odot}$ on the red giant branch before or right at core helium ignition (Saffer et al. 1994). One scenario for such extreme mass loss suggests that sdB stars lost significant envelope mass due to binary interactions on the red giant branch (Mengel, Norris, \& Gross 1976). If this were the sole channel for forming sdB stars, all sdB stars would be expected to be found in close binary systems - that is systems where the current separation is of the same order as the radius of the sdB progenitor star near the tip of the red giant branch, or $\mathrm{R} \approx 100 \mathrm{R}_{\odot} \approx 0.5 \mathrm{AU}$ (Maxted et al. 2001). Companions to sdB stars have been reported by numerous surveys, involving a variety of photometric and spectroscopic techniques, although these often have been of limited scope. These include: searches for flux excesses at long wavelengths, or flat energy distributions (e.g., Allard et al. 1987; Allard et al. 1994; Thejll, Ulla, \& MacDonald 1995; Ulla \& Thejll 1998); radial velocity variations (e.g., Maxted et al. 2001; Saffer, Green, \& Bowers 2001); the presence of a G-band, Ca II absorption, or other spectral features indicative of late type stars (e.g., Ferguson, Green, \& Liebert 1984; Bixler, Bowyer, \& Laget 1991; Theissen et al. 1995; Jeffery \& Pollacco 1998; Saffer, Green, \& Bowers 2001; Maxted et al. 2001); and even direct high-resolution imaging (e.g., Thejll, Theissen, \& Jimenez 1994; Heber et al. 2002).

Some of these survey reports have included an attempt to deduce the fraction of sdBs that are in binaries. The results have ranged from 50 to $100 \%$, depending on differing specific assumptions made about the nature and physical parameters of the sdB stars and/or their companions. For

\footnotetext{
${ }^{1}$ The term "extended horizontal branch" was coined by Greenstein \& Sargent (1974).
} 
example, the evolutionary status of the companion stars is typically not known. Some authors have preferred main sequence companions and less luminous sdB stars (i.e., Allard et al. 1994; Ferguson et al. 1984; Bixler et al. 1991), whereas others have preferred slightly evolved, sub-giant or giant companions and more luminous sdB stars (i.e., Jeffery \& Pollacco 1998; Theissen et al. 1995). The correction for "unseen" fainter companions (such as dM stars) in determining the total binary fraction is greatly affected by this choice. Thus, if the observed ( $\mathrm{G}-\mathrm{K}$ type) companions in composite systems are subgiants, the number of fainter unobserved companions (extrapolated using an assumed initial mass function) must be very large, and the resulting binary fraction is close to $100 \%$. On the other hand, if the $\mathrm{G}-\mathrm{K}$ companions are main sequence stars, the number of fainter unobserved companions is much smaller (because the lower inferred luminosity for the hot subdwarf is less effective at masking the light of such companions). The result is a binary fraction as low as $50 \%$. However, only the radial velocity or imaging studies give information about whether the sdBs that do have companions are in close binary systems.

The systematic survey of the entire sky that was carried out by the Two Micron All Sky Survey $^{2}$ (2MASS) provides the opportunity to further explore the question of whether all sdB stars (and sdO stars, to the extent that they represent the EHB) are in binary systems. This can be done by combining cataloged photometry at visible wavelengths with the 2MASS near-infrared imaging photometry. In addition to comparing visible and near-IR magnitudes, near-infrared colors for hot subdwarfs (e.g., $J-K_{S}$ ) can be used on their own as a second indicator of a binary system. In this paper we collect readily available visible and near-IR flux measurements of hot subdwarfs and identify those whose colors indicate the presence of a late type companion. We thus determine the fraction of subdwarfs that exist in composite spectrum binaries. (Note that not all classes of companion star will be identified this way, in particular white dwarf companions.) We extract visible photometry from an existing catalog of hot subdwarf stars, and we use IR photometry obtained from the 2MASS Second Incremental Data Release (2IDR) Catalog ${ }^{3}$. We look for IR colors that are "redder" than what would be expected for a normal hot subdwarf and which can be attributed to the presence of an unresolved late type companion. This method is similar to that used by Thejll et al. (1995) and Ulla \& Thejll (1998), but our sample is much larger and the IR photometry is more precise.

In $\S 2$ we describe our sample of hot subdwarfs including: improved coordinates, retrieval of 2MASS infrared photometry, availability and sources of visual photometry, and adopted classifications for objects in the initial sample. In $\S 3$ we examine and model a magnitude limited sample of hot subdwarfs using two color diagrams and the distribution in $J-K_{S}$ color. In $\S 4$ we introduce an approximately "volume limited" sample of hot subdwarfs and compare it to the magnitude limited sample. Finally, in $\S 5$ we summarize our findings.

\footnotetext{
${ }^{2}$ http://www.ipac.caltech.edu/2mass/

${ }^{3}$ http://www.ipac.caltech.edu/2mass/releases/second/index.html
} 


\section{The Sample}

For this investigation we studied hot subdwarf stars listed in the Catalogue of Spectroscopically Identified Hot Subdwarfs (Kilkenny, Heber, \& Drilling 1988) as updated and expanded in an electronic version by Kilkenny, c. 1992, kindly made available to us by D. Kilkenny. This catalog (hereinafter referred to as KHD) contains 1527 entries, of which 302 entries were added since the 1988 publication. Hot subdwarfs from the PG survey of UV-excess objects (Green, Schmidt, \& Liebert 1986) are an important component of the catalog (1017 objects with PG designations, either discovered or recovered by that survey). More recent or ongoing surveys, including the Hamburg Schmidt (HS) and Edinburgh-Cape (EC) surveys are not included. In the catalog sub-sample of 593 objects that is the subject of this paper, about $84 \%$ of the stars are to be found in the 1988 printed version of KHD, and about half of the sub-sample stars added to KHD between the 1988 and 1992 versions are from the Carnegie Southern Observatory H\&K survey (Beers et al. 1992). About $71 \%$ of the entries in KHD have northern Declinations, the same percentage as in the subsample studied here. The sub-sample is thus broadly representative of the parent catalog, for stars bright enough to be included in the 2MASS point source database. A number of important selection effects bias the parent catalog's contents, however, since it is compiled from many different sources each with its own criteria for inclusion or discovery (see also §3.3), but KHD is the most complete single compilation of field hot subdwarfs available, especially for the northern sky and for relatively bright objects.

While the KHD catalog contains all varieties of hot subdwarfs, we are primarily interested in the more numerous sdB stars. As mentioned above, sdB stars are understood to be relatively homogeneous and probably have a common evolution history from the zero age extended horizontal branch (ZAEHB) and beyond, while sdO stars likely follow multiple evolutionary pathways and might be expected to be less homogeneous and to have less simply explained properties. In the following sections, while we focus on the sdB stars, we will take the sdO stars "along for the ride" and comment on the two groups either separately ( $\mathrm{sdB}$ or sdO), or combined as "hot subdwarfs" in general (both $\mathrm{sdB}$ and $\mathrm{sdO}$ ). We further discuss the adopted classifications of objects from KHD and our treatment of them in $\S 2.4$.

\subsection{Coordinates}

To make a comparison of the KHD data with 2MASS, accurate coordinates on a consistent system are required. For each entry we verified the object's position by referring whenever possible to original published finding charts or by contacting knowledgeable observers, then locating the object on a chart prepared from the USNO A2.0 Catalog ${ }^{4}$ (Monet et al. 1996). We adopted coordinates from USNO A2.0, except for bright objects missing from that source, in which case

\footnotetext{
${ }^{4}$ http://www.nofs.navy.mil/data/fchpix/
} 
we used coordinates from the Tycho-2 (Høg et al. 2000) or Hipparcos (Perryman et al. 1997; ESA 1997) catalogs. These updated ICRS 2000.0 coordinates are accurate to a few arcseconds $\left(\sim 2^{\prime \prime}\right.$ or better) at the epoch of observation; in many cases this represents a very substantial improvement over the coordinates reported by KHD. During the time in which we collected the 2MASS data, we had confirmed and improved coordinates for 1459 objects (not counting 11 duplicate entries), while for 57 objects we had not yet positively identified the correct star. These remaining 57 unrecovered objects are not included in the present study. The full collection of improved coordinates will be published elsewhere (Wade \& Stark, in preparation).

\subsection{Infrared Photometry}

Infrared photometry $\left(J, H\right.$, and $\left.K_{S}\right)$ from the 2MASS 2IDR (which covers $\sim 45 \%$ of the sky) was collected by performing searches within $\sim 10^{\prime \prime}$ of the the updated coordinates. Visual verification was performed for crowded fields by comparing images from the Digitized Sky Survey ${ }^{5}$ (DSS) with images from 2MASS (most hot subdwarfs are uncrowded, with no other comparably bright field stars within $\sim 30^{\prime \prime}$ ). Objects are included in 2MASS if they have photometric uncertainty $\leq 0.155$ mag (signal-to-noise ratio, $\mathrm{S} / \mathrm{N} \geq 7$ ) in at least one of the three bands $\left(J, H\right.$, or $\left.K_{S}\right)$. This results in reliable photometry for point sources brighter than $J=15.8, H=15.1$, and $K_{S}=14.3^{6}$. $J-K_{S}$ errors for our objects are typically less than $\sim 0.2$ mag, with an average $J-K_{S}$ error of $\sim 0.12 \mathrm{mag}$ for our sample. Of the initial sample (1459 objects), we were able to retrieve 2MASS photometry for 571 hot subdwarfs (593 before removing 22 objects classified as non-subdwarfs). Of the 571 hot subdwarfs, only 385 had both $J$ and $K_{S}$ magnitudes listed. Of the other 186 objects, 180 have measurements of $J$ but only $95 \%$ confidence upper limits on $K_{S}$, and six have detections of a nearby companion to the subdwarf (but not the subdwarf itself). Also in the observations returned from 2MASS, a small fraction (few percent) of subdwarfs have two possible identifications within $\sim 10-20^{\prime \prime}$ of the input coordinates. The hot subdwarf members of these visual doubles were included in the present study since both finding charts and 2MASS images were available to identify the correct member of the pair. These visual doubles and their interpretation as possible physical binaries will be discussed in more detail in future work.

Table 1 lists the identifiers and coordinates for those objects from KHD that were found in 2MASS. Columns 1 and 2 list a sequence number ${ }^{7}$ and the first object identifier given in KHD. Columns 3 and 4 contain the J2000 coordinates based on the USNO A2.0 system. Column 5 is

\footnotetext{
${ }^{5}$ http://archive.stsci.edu/dss/index.html

${ }^{6}$ However, according to the Explanatory Supplement to the 2MASS Second Incremental Data Release (located at: http://www.ipac.caltech.edu/2mass/releases/second/doc/explsup.html), at high Galactic latitude, the 2MASS Point Source Catalog contains accurate detections 0.5-1.0 magnitudes fainter than these limits.

${ }^{7}$ The sequence number, assigned by us, is not intended to be used as a new designator in the literature, but is useful in navigating the KHD catalog and our Tables 1 and 2.
} 
the adopted classification for the hot subdwarf (see $\S 2.4$ ). Column 6 contains notes on individual objects as well as cross references for duplicate entries (see Table 1 footnotes). Column 7 lists an additional identifier for the hot subdwarf, if needed, using nomenclature consistent with $\mathrm{SIMBAD}^{8}$. For example, the designator CSHK 22876-35 used by KHD for star \#8 in this table is equivalent to the SIMBAD designator BPS CS 22876-35, and the designator KPD 00330+5229 used by KHD for star \#55 in this table is equivalent to the SIMBAD designator KPD 0033+5229. PG $23595+1942$ used by KHD for star \#1527 in this table is equivalent to the SIMBAD designator PG 2359+197. From now on, when we refer to a specific object from the sample, we will use both its sequence number (for ease in cross-referencing our Tables 1 and 2 with each other and with KHD) and a SIMBAD-compliant designator. If no other ID is given in the last column, the identifier from KHD (column 2) is consistent with SIMBAD. In this way, one SIMBAD-compliant designator for each star is available for cross-reference. The exceptions are the "HS" star \#390 HS 1000+4704 (Heber, Jordan, \& Weidemann 1991), the "SC" star \#1165 SC 1721-3338 from Reid et al. (1988), and the "LBQS" stars from Berg et al. (1992, \#41, 565, and 1405) which are not presently recognized by SIMBAD; we note that SIMBAD does use the LBQS designator for quasars from Hewett, Foltz, \& Chaffee (1995).

\subsection{Visual Photometry}

Visual photometry ( $B V$ or uvby) for the hot subdwarfs was taken from KHD, Allard et al. (1994), Landolt (1992), Mooney et al. (2000), Williams, McGraw, \& Grashuis (2001), and/or the Tycho-1 Catalog (Høg et al. 1998). For the photometry taken from KHD, when more than one set of photoelectric measurements was given, we favored Johnson $B V$ over Strömgren uvby, unless the only $B V$ measurement was reported as "uncertain" (then uvby was used). If only a single "uncertain" $B V$ measurement was available for an object (and no Strömgren data were found), then that uncertain measurement was nevertheless used and treated as exact. For consistency, we took the first of these measurements listed in KHD, unless it was uncertain, and even if additional measurements differed significantly from the first. Those hot subdwarfs with only photographic visual photometry were not included in the optical-infrared color space examination of hot subdwarfs discussed in $§ 3.1-3.2$ (however, those with 2MASS 2IDR detections are included in the objects discussed in §3.3). For stars listed in Allard et al. (1994), Landolt (1992), or Williams et al. (2001) the magnitudes from those sources were used in place of any reported in KHD. Strömgren uvby was taken from Mooney et al. (2000) for two objects: \#320 PG 09146+0004 and \#1327 PG 21325+1235 in preference to uncertain $V$ and no $B V$ (respectively) in KHD. Tycho-1 $B V$ magnitudes (Høg et al. 1998; ESA 1997) were taken for \#234 $\mathrm{BD}+34^{\circ} 1543$, which had only a single $V$ magnitude listed in KHD. No further literature search was undertaken to locate additional visual photometry. No errors are reported for the photometry given in KHD and we did not refer to original references to locate

\footnotetext{
${ }^{8}$ http://vizier.u-strasbg.fr/, operated at CDS, Strasbourg, France
} 
errors for each individual observation. However typical errors are $\lesssim 0.02$ mag for those surveys comprising the majority of the visual photometry (i.e., Allard et al. 1994; Green et al. 1986; Mooney et al. 2000; Wesemael et al. 1992; Williams et al. 2001) — thus, for simplicity, all measurements of visual photometry were treated as exact.

When only Strömgren photometry was available (or was taken in place of uncertain $B V$ ), we assumed that $y=V$, and $b-y$ was converted to $B-V$ using:

$$
(B-V)=1.584 \times(b-y)+0.681 \times m_{1}-0.116
$$

where: $m_{1} \equiv(v-b)-(b-y)$ (Turner 1990). Two objects have only synthetic $b$ and $y$ reported: \#1076 Balloon 083500007 and \#1095 Balloon 083600004. For these two objects, $B-V$ was estimated by setting $m_{1}=0$ in this equation.

Tycho-1 $B$ and $V$ magnitudes for one object, \#234 BD $+34^{\circ} 1543$, were converted to Johnson $B$ and $V$ magnitudes using:

$$
\begin{aligned}
V_{J} & =V_{T}-0.090 \times\left(B_{T}-V_{T}\right) \\
(B-V)_{J} & =0.850 \times\left(B_{T}-V_{T}\right)
\end{aligned}
$$

This conversion is valid over $-0.2<\left(B_{T}-V_{T}\right)<1.8$, and generally gives errors $<0.015$ in $V_{J}$ and $<0.05$ in $(B-V)_{J}($ ESA 1997).

Of the 571 reported hot subdwarfs found in the 2MASS 2IDR, we were able to collect photoelectric $V$ or $y$ magnitudes for 348 stars, and $B-V$ or the $b-y$ equivalent for 339 of these 348 stars.

All collected IR and visual photometry is presented in Table 2. Columns 1 and 2 list the sequence number and first identifier given in KHD. Columns 3-8 contain the 2MASS magnitudes $\left(J, H\right.$, and $\left.K_{S}\right)$ and their respective $1 \sigma$ errors. If no error is listed, then the star was not detected in that band, and the quoted magnitude is a 95\% confidence upper limit. Columns 9-10 are the calculated $J-K_{S}$ and combined $1 \sigma$ error for it. If no error is listed then the reported $J-K_{S}$ value is the maximum color index when an upper limit on $K_{S}$ is reported, and it is the minimum color index for an upper limit on $J$. Column 11 lists the $V, y$, or photographic visual magnitude of the hot subdwarf. Column 12 contains the $B-V$ color index for the hot subdwarf (derived from Strömgren uvby or Tycho-1 $B V$ in necessary, as discussed above). Column 13 is the adopted classification for the hot subdwarf (see $\S 2.4$ ). Column 14 contains additional notes, which are explained in the footnotes to Table 2.

\subsection{Classifications}

During our analysis, we divided the total sample of hot subdwarfs with 2MASS 2IDR data into two sub-samples: "sdB/sd" and "sdO/sdOB" based on their classifications in KHD. For consistency, 
we took the first classification given, even if there were additional conflicting classifications. Our "sdB/sd" group comprises those objects classified as sdB, sdB-O, and sd. Our "sdO/sdOB" group comprises $\mathrm{sdO}, \mathrm{sdO}(\mathrm{A}), \operatorname{sdO}(\mathrm{B}), \mathrm{sdO}(\mathrm{C}), \mathrm{sdO}(\mathrm{D})$, and $\mathrm{sdOB}$. There is ongoing discussion in the community regarding appropriate multi-dimensional classification schemes for hot subdwarf stars, which likely will depend on the spectral resolution used (Jeffery et al. 1997; Drilling et al. 2000, 2003). Meanwhile, we have made no attempt to improve upon, regularize, or rationalize the various classifications summarized in KHD.

As noted earlier, 22 additional objects were excluded from our sample because they are not subdwarfs. These objects are classified: HBB (three objects; Tables 1 and 2 sequence \#: 350, 642, and 662), DA (two; \#265 and 588), non-sd (nine; \#269, 287, 574, 676, 734, 794, 1138, 1443, and 1501), only as "bin?" (three; \#732, 1076, and 1095), or had no classification (four; \#398, 702, 884, and 967) in KHD. Lastly, \#276 KUV 08388+4029 is listed as "sdB" in KHD, however, SIMBAD lists this object as a Seyfert 1 galaxy at $z \approx 0.15$. We verified its AGN nature with a low-resolution spectrum obtained from the Hobby-Eberly Telescope ${ }^{9}$, which clearly showed the $\mathrm{H} \alpha$ and $\mathrm{H} \beta$ broad emission lines at wavelengths consistent with a redshift $z \approx 0.15$.

Hereafter, when we refer to "sdB stars" we mean our "sdB/sd" group, when we refer to "sdO stars" we mean our "sdO/sdOB" group, and when we refer to "hot subdwarfs" we mean the entire sample ( $\mathrm{sdB}$ and $\mathrm{sdO}$ ) excluding the non-subdwarfs just mentioned. A summary of the numbers and types of subdwarfs at each stage in the data gathering process is presented in Table 3.

\section{Hot Subdwarfs in Color Space}

\subsection{Optical-IR Color-Color Plots}

After examining various combinations of color indices, we chose to focus on $\left(J-K_{S}\right)$ vs. $(B-V)$ and $\left(J-K_{S}\right)$ vs. $\left(V-K_{S}\right)$. These two combinations allow us to extract most of the information from the available photometry. Substituting $J-H$ in place of $J-K_{S}$ shows the same features and trends. Likewise, substituting $V-J$ for $V-K_{S}$ shows the same features and trends. Using $H-K_{S}$ did not provide any additional information.

The locations of single and composite hot subdwarfs in color space were determined by selecting samples of hot subdwarfs reported as single and composite from the literature. A total of 69 reportedly single stars and 37 reported composites that had 2MASS detections were taken from the literature (KHD; Ferguson et al. 1984; Beers et al. 1992; Allard et al. 1994; Thejll et al. 1995; Ulla \& Thejll 1998) or a list of hot subdwarfs spectroscopically observed and kindly provided to us by

\footnotetext{
${ }^{9}$ The Hobby-Eberly Telescope is operated by McDonald Observatory on behalf of The University of Texas at Austin, the Pennsylvania State University, Stanford University, Ludwig-Maximilians-Universität München, and Georg-August-Universität Göttingen. The Marcario Low Resolution Spectrograph is a joint project of the HobbyEberly Telescope partnership and the Instituto de Astronomía de la Universidad Nacional Autonoma de México.
} 
E. M. Green. After removing from this list stars with incomplete photometry and stars classified as "non-sd" by KHD, 33 (24 sdB, $9 \mathrm{sdO})$ of the reported composites and 59 (48 sdB, $11 \mathrm{sdO})$ of the reported singles remained and are used here to determine the composite and single loci (these stars are flagged in Table 2 with a " $s$ " or " $c$ " in the last column).

When plotted in $\left(J-K_{S}, B-V\right)$ or $\left(J-K_{S}, V-K_{S}\right)$ color spaces relative to the location of the Pop I main sequence (Figure 1), the reported single hot subdwarfs (both sdB and sdO) occupy the same area in color space as other $\mathrm{O}$ and $\mathrm{B}$ stars, with the sdOs in general having slightly bluer colors than the sdBs. Most of the single hot subdwarfs occupy the region: $(B-V) \lesssim+0.1$, $\left(V-K_{S}\right) \lesssim+0.2$, and $\left(J-K_{S}\right) \lesssim+0.05$. This region is marked by a box on Figure 1 . On the other hand, reported composite hot subdwarfs (both sdB and sdO) occupy a separate and distinct region of color space; they have redder colors than single subdwarf stars. Composite subdwarfs show a consistent and quite large color difference from the "typical" single subdwarf in $J-K_{S}$. The difference in $V-K_{S}$ is also large in the mean, but the distributions of single and composite systems overlap slightly. The least average difference is in $B-V$ (Figure 1).

These patterns can be understood if the hot subdwarf's companion star in the composite systems is cool and relatively faint at visible wavelengths. The bluest color index, $B-V$, will be relatively unaffected by the additional light from the companion. The visual-infrared index, $V-K_{S}$, will betray a large infrared "excess" compared to a single hot subdwarf, but there will be a large spread because the index depends on the competing light from the two stars at $V$. The pure infrared index, $J-K_{S}$, will be dominated by the cool companion, whose color is very different from that of the hot star.

Three reported composite sdOs have distinctly different colors in Figure 1 compared with all of the other reported composites. The first (labeled "1" in Figure 1) is \#1355 BD $-3^{\circ} 5357$ (or FF Aqr, with $B-V=+0.895, J-K_{S}=+0.752$, and $V-K_{S}=+2.838$ ), an eclipsing binary (orbital period $\sim 9.2$ days) classified sdOB+G8III (Dworetsky et al. 1977; Etzel et al. 1977). The second ("2") is \#1457 BD $-7^{\circ} 5977$ (with $B-V=+0.541, J-K_{S}=+0.569$, and $V-K_{S}=+2.129$ ), classified sdOB+K0IV-III by Viton et al. (1991). The third ("3") is \#127 PG 0205+134 (with $B-V=+0.006, J-K_{S}=+0.847$, and $\left.V-K_{S}=+2.771\right)$, classified "sdOB comp" by Green et al. (1986), and further refined to sdOB+M3.5(IV?) by Allard et al. (1994) and Williams et al. (2001). All of these cool companions are atypically luminous for sdO-type composites, which otherwise have main sequence or subgiant companions reported, so it is not unreasonable for these three objects to have exceptionally red colors.

The two sdB stars labeled "4" (\#822 PG 1439-013) and "5" (\#744 TON 183) in Figure 1, have anomalously blue $J-K_{S}$ colors, $J-K_{S}=-0.496 \pm 0.148$ and $-0.494 \pm 0.090$ respectively ( $1 \sigma$ errors). These colors could be due to photometric errors, or they could be intrinsic to the objects themselves. The color index $J-H$ generally shows the same trend as $J-K_{S}$, and these two stars have essentially the same $J-H$ colors as all other single sdBs $(J-H=-0.097$ and -0.196$)$. Possibly, these unusual $J-K_{S}$ colors are due to photometric errors in $K_{S}$. 
One reported single sdB star, \#483 TON 64 (labeled "6" in the right plot of Figure 1), has a rather red value for $V-K_{S}(+0.592)$, yet appears "normal" in the other colors $(B-V=-0.220$, $\left.J-K_{S}=-0.028\right)$. Its unusual position may be due to an error in the standardization of the $V$ magnitude. The two measurements for the visual magnitude of this star in KHD are discrepant: $V=15.86$ and $y=14.59$. Our adopted procedure is to favor $V$ over $y$; if however we were to use $y=14.59$ in place of $V=15.86$, we would have $V-K_{S}=-0.678$ (again assuming $y-K_{S}=V-K_{S}$ ), which places this star near the center of the single subdwarf locus. Nevertheless for consistency, we continue to use the value of $V$ for this object.

Now placing all of the 277 hot subdwarfs with $V(273$ with $B-V)$ and $J$ and $K_{S}$ measurements in color space (Figure 2), we observe that they occupy the same areas in color space as defined by the smaller sample of known composites and singles. (Objects with only upper limits on $K_{S}$ from 2MASS are not plotted in Figure 2.) There is some additional scatter, which is most likely due to errors in the photometry. This scatter could, in principle, also be due in part to differences in interstellar extinction and reddening from object to object. Additionally, some hot subdwarfs are known to pulsate such as the V361 Hya stars ${ }^{10}$ (O'Donoghue et al. 1999) and the "PG 1716" stars (Green et al. 2003). In the case of the V361 Hya stars, the pulsation amplitudes are generally less than $0.01 \mathrm{mag}$ and typically only a few milli-mags with typical periods of 100-250 seconds (i.e., Kilkenny et al. 1997; Østensen et al. 2001; Green et al. 2003). The "PG 1716" stars have peak-to-peak amplitudes $\lesssim 0.05$ magnitudes over timescales of about an hour (Green et al. 2003). Light variations due to both of these pulsations are typically less than other sources of error.

Figure 2 suggests that candidates for composite systems (hot subdwarf+late type star) can be identified and targeted for future study using $B-V$ or $V-K_{S}$ in combination with $J-K_{S}$. Unfortunately, in order to use this technique, accurate visual photometry is needed in addition to IR photometry.

\subsection{Modeling the Distribution in Optical-IR Color-Color Space}

To test the interpretation offered above, that the colors of single and composite hot subdwarfs may be understood if the companions are cool stars, we attempted to reproduce the location of composite subdwarfs in color space by combining the colors of a typical sdB with those of late type Pop I main sequence stars. Color indices for late type stars were taken from Johnson (1966); for this exercise we assumed $K \approx K_{S}$. The "typical" colors for sdBs were taken from the center of the clump of reported single stars in Figure 1 . We used $(B-V)_{\mathrm{sdB}} \approx-0.25,\left(V-K_{S}\right)_{\mathrm{sdB}} \approx-0.75$, $\left(J-K_{S}\right)_{\mathrm{sdB}} \approx-0.15$. Based on the spread seen in Figure 1, however, it is likely that single sdB stars in fact have spreads in color index on the order of $\Delta(B-V) \sim \pm 0.1, \Delta\left(V-K_{S}\right) \sim \pm 0.2$, and $\Delta\left(J-K_{S}\right) \sim \pm 0.15$, as might be expected from variations in the physical properties of the sdBs

\footnotetext{
${ }^{10}$ also known unofficially as "EC 14026 " stars
} 
(most notably the effective temperature); some of the spread in $V-K_{S}$ and $J-K_{S}$ is however due to limited IR photometric precision.

The colors were combined, varying the fraction of the light at $V$ that arises from the companion. The resultant locus passes among the known composite sdBs with contributions from the companion of $\sim 10-60 \%$ at $V$, and spectral types G-K (Figure 3 ). These fractional contributions and companion types agree with those reported by Allard et al. (1994) for sdBs, and Ferguson et al. (1984) and Orosz, Wade, \& Harlow (1997) for hot sdBs and sdOs. While this general analysis also fits the sdOs, the unreddened colors of a "typical" sdO should be somewhat bluer than those of a sdB, since sdOs are hotter than sdBs.

The loci are plotted with the entire subdwarf sample in Figure 4.

By introducing assumed absolute magnitudes along with colors, we can synthesize composite colors specifically for sdB+late type main sequence stars. We took colors and $M_{V}$ for Pop I main sequence stars from Johnson (1966) and Drilling \& Landolt (2000) and assumed colors and $M_{V, \mathrm{sdB}}$ for the sdB component. The sdB colors used in the previous calculation were again adopted. The absolute magnitudes of sdB stars are poorly constrained, with reported values spanning the range from $M_{V \text {,sdB }} \approx 3.5-6.2$ (Allard et al. 1994; Bixler et al. 1991; Ferguson et al. 1984; Liebert, Saffer, \& Green 1994; Moehler, Heber, \& de Boer 1990; Orosz et al. 1997; Thejll et al. 1997). We therefore treated $M_{V, \mathrm{sdB}}$ as a parameter, and varied it (choosing $M_{V, \mathrm{sdB}}=3.5,4.0,4.5,5.0$, and 5.5) to see its effect on the location of the synthesized composites in color space (Figure 5). We found that the locus of synthetic composites passes among the observed composite systems and best characterizes the observed companions as having Pop I spectral types $\mathrm{G}-\mathrm{K}$ when $M_{V \text {,sdB }} \approx 4.5-5.0 \mathrm{mag}$ (if the companions lie on the main sequence). It should be noted, however, that the same pattern of color loci can be created by fixing $M_{V, \mathrm{sdB}}$ and instead varying the absolute magnitudes of the companions from their main sequence values. If $\mathrm{G}-\mathrm{K}$ type companions were subgiants, they would be more luminous by perhaps $\sim 2$ magnitudes, and would therefore give the same pattern with a more luminous sdB, $M_{V \text {,sdB }} \approx 2.5-3.0 \mathrm{mag}$ (but we would not expect to see evolved M-type companions). In nature, there is likely to be some range in absolute magnitude for both the sdB stars and their companions. (In addition to the variations of luminosity and bolometric correction along the zero-age EHB, sdB stars should evolve to higher luminosity on timescales of $10^{8}$ years.)

The (main-sequence) loci are plotted with the entire hot subdwarf sample in Figure 6.

These simple models give results consistent with previous studies concerning the companion spectral types and their light contributions. We cannot, however, assume an $M_{V}$ for the companion and invert the calculation case-by-case to give reliable individual values for $M_{V \text {,sdB }}$, nor vice versa, because of the uncertainties in the measured magnitudes, colors, and reddening. Additionally there is the expected intrinsic spread in $M_{V \text {,sdB }}$ referred to above (for further discussion see Saffer et al. 1994). For similar reasons, neither can we infer exact companion spectral types for individual objects.

Of the 33 currently known composite hot subdwarf systems, essentially all of the cool com- 
panion stars for which a spectral class is known are of spectral types G or K. Our models show that composite systems with G, K, and early M-type companions show the most significant differences in color from a single sdB (particularly in $J-K_{S}$ ) and from main sequence stars. This could explain why most known composite systems have GK-type companions: for companions earlier then $\sim \mathrm{G}$, the combined system looks more like a main sequence star (or a cool, Pop II subdwarf) than a sdB star. Such systems would likely be excluded from catalogs of hot subdwarfs. For main-sequence companions much later than $\sim \mathrm{M} 0$, the effect on the combined system light is so small that the companion would not easily be detected in the visual. Most of the spectroscopic studies of composite sdBs have been done in the visual and blue region of the spectrum where very little color difference between a composite $\mathrm{sdB}+\mathrm{dM}$ and a single sdB exists, and there are indeed few identifications of dM companions - thus far there are only four known: PG 1241-084 (also HW Vir; Menzies \& Marang 1986), PG 1336-018 (Kilkenny et al. 1998), HS 0705+6700 (Drechsel et al. 2001), and PG 1017-086 (Maxted et al. 2002). All of these are very short period binaries $\left(2^{\mathrm{h}} 48^{\mathrm{m}}, 2^{\mathrm{h}} 25^{\mathrm{m}}, 2^{\mathrm{h}} 18^{\mathrm{m}}\right.$, and $1^{\mathrm{h}} 45^{\mathrm{m}}$ respectively), and the the nature of the companion was studied in part by eclipses or reflection effects in the light curve. PG 1241-084 (KHD sequence \#605) and PG 1017-086 (\#407) are found in the 2MASS 2IDR. Colors for such close binary stars, constructed from non-simultaneous measurements of visible and infrared magnitudes, may be unusual. The color indices for these two stars are nevertheless consistent with single hot subdwarfs (\#605 PG 1241-084: $J-K_{S}=-0.149, B-V=-0.332, V-K_{S}=-0.110 ; \# 407$ PG 1017-086: $\left.J-K_{S}=-0.176, B-V=-0.225, V-K_{S}=-0.417\right)$. A few other objects can be noted in the region of color space that, based on our simple models, would be occupied by sd+dM composites (Figures 4 and 6). Owing to uncertainties in the measured magnitudes and colors, however, we cannot clearly distinguish early $\mathrm{M}$ companions from late $\mathrm{K}$ companions - near-IR or IR spectroscopy will be needed to distinguish clearly between late $\mathrm{K}$ and early M-type companions. Yet it seems clear, based on our color plots, that there are not great numbers of "hidden" early M-type ( M0) companions to hot subdwarfs. For main sequence companions substantially later than $\sim \mathrm{M} 0$, the $J-K_{S}$ color is rapidly returning to that of a single subdwarf.

Identifying and characterizing further M-type companions to sdB stars would help settle some of the questions about the nature of earlier-type $(\mathrm{G}-\mathrm{K})$ companions, in particular whether they are predominately main sequence or subgiant stars. Late M-type companions can safely be assumed to be on the main sequence. If many of these are found among the sdB stars with "composite" colors, the relatively faint $M_{V \text {,sdB }}$ inferred from such systems would imply that the $\mathrm{G}-\mathrm{K}$ type companions in other composite-color systems are also close to the main sequence. If late-M companions are not found in great numbers, it could be inferred that the $\mathrm{G}-\mathrm{K}$ companions are mostly subgiants, since the corresponding bright $M_{V \text {,sdB }}$ inferred from this assumption would explain the absence of $\mathrm{sd}+\mathrm{dM}$ systems detectable from photometry. The existence of many subgiant companions, with their short lifetimes as subgiants, would be interesting, since the evolutionary timescale of sdB stars is also short. 


\subsection{Hot Subdwarf Distribution in $J-K_{S}$ Color}

Since visual photometry is not available for all of the hot subdwarfs contained in the 2IDR, and since there is a more distinct separation between composites and single subdwarfs stars in $J-K_{S}$ than in the other colors, we examined the distribution of subdwarfs based on their $J-K_{S}$ colors alone. A histogram of $J-K_{S}$ for all subdwarfs with $J$ and $K_{S}$ measurements (385 subdwarfs: 294 $\mathrm{sdB}, 91 \mathrm{sdO}$ ), reveals a bimodal distribution (Figure 7 ). One peak is centered near $J-K_{S} \approx-0.15$ ( $\sim \mathrm{B} 2-\mathrm{B} 3$ spectral type), and the other is centered near $J-K_{S} \approx+0.30$ ( $\sim$ G5 spectral type). There are also wings stretching to both redder and bluer colors. This distribution cannot be explained by reddening, because no significant trends were observed with galactic latitude. We would also expect reddening to produce a tail to the red from a single peak, but not a pronounced second peak. Finally, the amount of extinction that would be required is also excessive.

We sorted the sdB and $\mathrm{sdO}$ samples into three groups by $J$ magnitude. The bimodal peaks are very pronounced in the brightest third of each sample, less distinct in the middle third, and practically non-discernible in the faintest third. This pattern can be fully explained by the increase in photometric uncertainty at fainter magnitudes.

In keeping with the results found from our modeling of color-color distributions, we postulate that the stars within the blue peak of the $J-K_{S}$ distribution $\left(J-K_{S}<+0.05\right)$ are single stars (or $\mathrm{sd}+\mathrm{WD}$ pairs, with colors indistinguishable from single hot subdwarfs), and that stars in the red

peak $\left(J-K_{S}>+0.05\right)$ are composite (sd+late type) systems. The color index separating these two groups of stars was adopted from the box defining the region of single subdwarfs in Figure 1. With this definition, we find that $58 \pm 5 \%$ of the subdwarf stars in our 2MASS 2IDR sample are apparently single, and $42 \pm 5 \%$ are composite. The quoted $95 \%$ confidence errors are sampling errors computed from the binomial distribution, taking the size of the sample into account. If the color index of the boundary separating single and composites is increased (decreased) by $0.05 \mathrm{mag}$, the fraction of subdwarfs that are "blue" (i.e., single) is increased (decreased) by $3 \%(3 \%)$.

While the 2IDR sample is representative of the KHD catalog as a whole, it is likely that neither of these is representative of the true hot subdwarf population in the solar neighborhood. There are the usual completeness issues, including heterogeneous magnitude limits and different classification criteria used by the sources from which KHD was compiled (although we note that about $2 / 3$ of the cataloged subdwarfs are drawn from the PG survey, Green et al. 1986, which has rather well-defined properties). We draw renewed attention, however, to a kind of censorship bias that is present in the PG survey. An unknown number of composite subdwarfs may have been excluded "by design" (i.e., not included in the PG catalog and never identified as hot subdwarfs), since according to Green et al. (1986) UV excess stars were omitted from their catalog if their spectra showed a pronounced Ca II K line. (The supposition was that many of these would be metal-weak F or G subdwarfs, which met the purely photometric criterion for inclusion as UV excess stars owing to a combination of reduced line blanketing and larger than average photometric errors.) Thus composite hot subdwarf systems with $\mathrm{F}$ or $\mathrm{G}$ companions might be underrepresented in KHD and the present sub-sample 
drawn from it. (On the other hand, since some PG subdwarfs are found in other independent surveys, some of the composite systems "missing" from PG may nevertheless have been included in the KHD compilation, from other sources.) The number of "excluded" objects is roughly 1000-1200 (R. F. Green 2003, private communication), but the fraction of these that may be composite hot subdwarfs rather than $\mathrm{G}$ subdwarfs is not known, so these selection effects are difficult to quantify. In time, objective prism-based surveys such as the one being carried out with the Hamburg Schmidt telescope may provide a catalog of hot subdwarfs that is more clearly representative of the Galactic population. Meanwhile, we proceed to analyze the 2MASS 2IDR sample from the KHD catalog. It must be realized, however, that since the 2MASS 2IDR represents a magnitude limited survey, the composite systems (being more luminous than single subdwarfs) will be overrepresented in this sample, other things being equal (see $\S 4$ for further discussion of this point).

The same bimodal distribution in $J-K_{S}$ exists in the sub-sample of sdB/sd stars that is found in the full sample $\left(58 \pm 6 \%\right.$ have $J-K_{S}<+0.05$, and $42 \pm 6 \%$ have $\left.J-K_{S}>+0.05\right)$. The sub-sample of sdO/sdOB stars shows a less clear pattern. There is a hint of three groups: one near $J-K_{S} \sim-0.2$, another near $J-K_{S} \sim+0.2$, and the third near $J-K_{S} \sim+0.5$, but the small number of stars in each group makes this finding quite tentative. The two larger groups of sdO stars $\left(J-K_{S} \sim-0.2\right.$ and +0.2$)$, if real, can be considered to fall to the "blue" side (lower $\left.J-K_{S}\right)$ of the the two peaks in the sdB distribution, consistent with the higher temperatures of the sdO with respect to the sdB stars. Overall, the observed bimodal distribution in $J-K_{S}$ of all hot subdwarfs is defined by the majority sdB stars. The fraction in each peak of the $J-K_{S}$ distribution does not significantly change if the sdO stars are removed. A breakdown of the fractions of subdwarfs by $J-K_{S}$ color is shown in Table 4 .

\section{4. $\quad$ Fitting the sdB $J-K_{S}$ Distribution}

We have fitted the bimodal $J-K_{S}$ distribution of the sdB stars (alone) to the sum of two Gaussians, centered on the blue and red peaks. An initial "fit-by-eye" to the binned data was improved by minimizing $\chi^{2}$ on a range that was trimmed $\left(-0.45<J-K_{S}<+0.6\right)$ to remove the effects of the reddest and bluest outliers. The $\chi^{2}$ minimization was done using a systematic gridded search in the neighborhood of the initial fit. There are six fitting parameters: two mean values (or centers), two dispersions (or widths), and two normalizations (or amplitudes). The final fit has $\chi^{2}=6.8$ (reduced $\chi_{\mathrm{R}}^{2}=0.45$, with 15 degrees of freedom). The parameters for the fit are listed in Table 5 and the fit is shown in Figure 8.

While the $\chi^{2}$ statistic provides a useful criterion for improving the fit, its final numerical value should be used with caution, since the range of the fit was trimmed, and since some of the data bins contain only a few members. In particular, the $95 \%$ confidence intervals shown in Table 5 are indicative rather than definitive. The small value of $\chi_{\mathrm{R}}^{2}$ nevertheless demonstrates the plausibility of a two-Gaussian fit, which is confirmed by visual inspection. The "blue" Gaussian has a somewhat smaller Gaussian dispersion than the red one, but both dispersions $(\sigma \approx 0.09,0.16)$ are close to the 
average photometric error for this sample from $2 \mathrm{MASS}, \sigma\left(J-K_{S}\right) \approx 0.12$. This makes it difficult to state whether there is any intrinsic spread in the $J-K_{S}$ colors of the single or composite sdBs.

To the extent that the separation of single and composite sdB stars in the sample is accurately represented by two Gaussians, it is seen that there is very little overlap in $J-K_{S}$ color index between the two groups of stars. The areas of the two Gaussians are in the proportion 57:43, consistent with the binomial result found using a sharp demarcation at $J-K_{S}=0.05$.

\section{A "Volume Limited" Hot Subdwarf Sample}

\subsection{Defining the Sample}

There is a strong advantage in working with the $J-K_{S}$ color index to discuss the fraction of composite-color binaries among hot subdwarfs, as in $\S 3.3$ and 3.4 above, since the data are available for a larger sample than would be the case for optical or mixed optical-infrared colors. Also, the $J$ and $K_{S}$ from 2MASS are homogeneous in nature. However, a strong selection bias in these data exists, because 2MASS itself is an approximately magnitude limited survey (albeit with different magnitude limits in each of three passbands). The entire sample of hot subdwarfs we have been discussing (common to both KHD and 2MASS 2IDR) is thus a magnitude limited sample in some sense. The volume sampled for single subdwarfs is therefore smaller than that sampled for composites, since the presence of the late type companion increases the distance at which a composite system remains brighter than the limiting 2MASS magnitude.

To further explore this selection bias, we constructed $\left(J, J-K_{S}\right)$ and $\left(K_{S}, J-K_{S}\right)$ colormagnitude diagrams for the full 2MASS 2IDR sample (Figure 9). Several interesting patterns are noted. First, the two populations (single and composite) can be seen, and both are observed to cover the entire magnitude range (i.e., composites and/or singles are not "clumped" at a specific apparent magnitude). Second, a majority of the objects with extreme $J-K_{S}$ colors (either blue or red) are found at the faintest magnitudes, an effect largely explained by photometric errors. Third, it can be seen that the sample is limited by detections in the $K_{S}$ band. There is a clear cut-off at $K_{S} \approx 15.75$, while in $J$ the cut-off magnitude varies by color (redder objects that are detected in both $J$ and $K_{S}$ run to a fainter $J$ magnitude than the bluer objects).

The interpretation of this third feature is that a blue (single) object detected near $J=15.5$ or fainter is missing at $K_{S}$ and thus is not part of the plotted sample; a red (composite) object at the same $J$ will also have a $K_{S}$ detection and thus will appear in Figure 9. (This effect is distinct from the fact that composites appear brighter at $J$ due to the combined light from two stars.)

Recognizing this selection bias, we can attempt to define an approximately volume limited sample of hot subdwarfs. (To our knowledge, this is the first time that this bias has been discussed in the context of hot subdwarf composites. Theissen et al. (1992) discussed a "statistically complete" sample containing 11 hot subdwarfs, however they did not address the composite vs. single bias.) 
To define our volume limited sample, we try to remove those subdwarfs that we observe to be composite, but which would not have been detected by 2MASS had they been single. With only a single color index to work with, and with uncertainties in both the photometry and the relative luminosities of the hot subdwarfs and their companions, we cannot rely on such a technique to say whether any given individual system lies within the defined volume. The sample so constructed can be used in a statistical sense, however, to reflect the general nature of the true distribution, and at least to indicate the size of the selection effect.

The observed cut-off magnitude from Figure 9 for a single hot subdwarf is $K_{S} \approx 15.75$. Taking this limit as the apparent magnitude of the hot subdwarf component in a composite system, we can compute the corresponding apparent magnitude for the total light from the system, using the models discussed in $§ 3.2$ and shown in Figures 3 and 4 . This total magnitude will vary as the spectral type and/or relative luminosity of the late type companion is varied. The locus of thuscomputed magnitudes is shown in the lower panel of Figure 9 for a hot subdwarf with the "typical" color $J-K_{S}=-0.15$ adopted in $\S 3.2$, for companions with colors of the Pop I main sequence spectral types G0, K0, K5, and M0. Along each of these lines, the color and total magnitude varies as the relative brightnesses of the two component stars are varied. Note that no assumption has been made about the absolute magnitude of either the hot subdwarf or the cool companion.

We now replace the family of lines (corresponding to different spectral types of the cool star) with a single representative line. (This is because based on $J-K_{S}$ alone we cannot know the true spectral types of the late type companions without making assumptions about the relative luminosities of the component stars.) We use this line to approximate the appropriate magnitude

limit for a volume limited sample, again in a statistical sense. The line is chosen to pass though the middle of the known composite sdBs shown in Figure 3; it thus represents a $\sim 5 \%$ contribution (at $V$ ) by a K5 star, or a contribution of $40-80 \%$ at K0 in Figure 9 . The change in the volume-limiting $K_{S}$ magnitude is thus most rapid near $J-K_{S} \approx+0.3$ (see Figure 3). All hot subdwarf stars in the $\left(K_{S}, J-K_{S}\right)$ color-magnitude diagram that fall above this line are included in our statistical volume limited sample. The resulting sample size is 300 hot subdwarfs ( $228 \mathrm{sdB}$ and $72 \mathrm{sdO}$ ). The average error in $J-K_{S}$ for this new sample is 0.10 mag (slightly reduced from 0.12 mag, which applies to the full $J-K_{S}$ sample).

\subsection{Color-Color and $J-K_{S}$ Distribution of the Volume Limited Sample}

For the volume limited sample, visual magnitudes ( $B V$ or the uvby equivalent) are available for $176 \mathrm{sdB}$ stars (4 additional with $V$ only) and $61 \mathrm{sdO}$ stars ( 1 additional with $V$ only). The volume limited sample occupies the same regions of $\left(J-K_{S}, B-V\right)$ and $\left(J-K_{S}, V-K_{S}\right)$ color spaces (Figure 10) that the magnitude limited sample does (compare Figures 2 and 10), but many of the more extreme red objects (in $J-K_{S}$ ) have been removed.

The $J-K_{S}$ histogram made using only the volume limited sample (Figure 11) can be compared 
with the corresponding diagram for the entire magnitude limited sample (Figure 7 ). The distribution is still bimodal, with one peak centered near $J-K_{S} \approx-0.15$ as before $(\sim \mathrm{B} 2-\mathrm{B} 3$ spectral type), and the other near $J-K_{S} \approx+0.30$ ( $\sim$ G5 spectral type). The sdB stars still dominate the total hot subdwarf distribution, and the sdO stars show a stronger hint of bimodality (again with both peaks shifted slightly blueward of the sdB peaks). The blue wing to the distribution remains, while the red wing has been greatly reduced. Most important, the fractions of single ("blue") and composite ("red") systems have been altered (Table 6). Whereas the magnitude limited sample showed a 58:42 split between single and composite sdB stars (Table 4), the volume limited sample shows a 75:25 split in favor of single sdB stars. Likewise, the split for the total hot subdwarf sample is 73:27 in the volume limited sample (as opposed to 58:42 in the magnitude limited sample).

We can again describe the $\operatorname{sdB} J-K_{S}$ distribution for the volume limited sample as the sum of two Gaussians. The new $\chi^{2}=8.9$ (reduced $\chi_{\mathrm{R}}^{2}=0.59$, with 15 degrees of freedom). The parameters for the fit are given in Table 7, and the fit is shown in Figure 12.

The parameters of the blue Gaussian fits for the magnitude limited and volume limited samples are essentially the same. This follows because the number of stars that fall in the blue peak was not substantially altered in creating the volume limited sample. The width of the blue Gaussian is consistent with the average photometric error from 2MASS for this sample, $\sigma\left(J-K_{S}\right) \approx 0.10$, implying that there is little intrinsic spread in the $J-K_{S}$ color index of single sdB stars.

The red Gaussians for the magnitude limited and volume limited samples have different mean values (centers), with the volume limited sample shifted blueward relative to the magnitude limited sample $\left[\Delta\left(J-K_{S}\right) \approx-0.04\right]$. The normalizations (amplitudes) are different, owing to to the decrease in the number of red stars in the volume limited sample. The change in center is likely

due to the removal of the reddest and also the faintest (and thus most uncertain) measurements from the red peak. The dispersion of the red Gaussian that represents the volume limited sample, $\sigma\left(J-K_{S}\right) \approx 0.10$, is reduced from the magnitude limited case, and is now consistent with the 2MASS photometric error for this new sample $(\approx 0.10 \mathrm{mag})$; this implies that there is little variation in the $J-K_{S}$ color of the remaining composite systems.

\section{Summary}

Using updated and improved coordinates for hot subdwarfs cataloged by KHD, we have retrieved 2MASS 2IDR photometry for 571 hot subdwarfs. This is the largest sample of hot subdwarfs, and in particular composite systems, ever examined. We divided the sample into two sub-samples based on their classifications in KHD: sdB/sd ("sdB" group), sdO/sdOB ("sdO" group). We examined these two sub-samples both independently and combined as a whole. We examined the distribution of hot subdwarfs in optical-IR color-color plots, by combining 2MASS with visual photometry ( $B V$ or $u v b y$ ) from KHD (or a few selected other sources). The most revealing combinations of color indices we found were: $\left(J-K_{S}, B-V\right)$ and $\left(J-K_{S}, V-K_{S}\right)$. We also examined the 
distribution of hot subdwarfs in $J-K_{S}$ only and found a bimodally distributed population. Finally, we attempted to define an approximately volume limited sample of hot subdwarfs for statistical purposes. This is the first time (to our knowledge), that the selection bias favoring composite hot subdwarfs in magnitude limited surveys has been discussed, and an approximately volume limited statistical sample defined.

In keeping with the exploratory nature of this work and the large sample size, we have favored a statistical approach, in contrast to the attempt to find a best characterization of individual binaries. (The relatively large mean errors of the 2MASS photometry also figured in the decision to use this approach.) In the same spirit, we have contented ourselves with "naive" modeling of the colors, e.g., adopting single values for the colors and magnitudes of the subdwarfs rather than a distribution of these quantities - our goal in the modeling is to support the overall division of the sample of stars into single and composite groups, by demonstrating the plausibility of such modeling.

We found that the locations of hot subdwarfs in visual-IR color-color diagrams can be modeled (very simply and without assuming luminosities for either the hot subdwarf or the companion) as either a single B-type star or as a combination of two stars (spectral type B representing the hot subdwarf, and spectral type $\mathrm{G}-\mathrm{K}$ for a companion) with contributions from the late type companion of $\sim 10-60 \%$ at $V$. To a first approximation sdO stars can be described in the same manner as $\mathrm{sdB}$ stars. If main sequence companions are adopted, then the sdB stars are best described with $M_{V, \mathrm{sdB}} \approx 4.5-5.0 \mathrm{mag}$, and they typically have $\mathrm{G}-\mathrm{K}$ type companions. However, the observed distribution can be reproduced equally well by assuming subgiant companions with more luminous $\mathrm{sdB}$ stars $\left(M_{V, \mathrm{sdB}} \approx 2.5-3.0 \mathrm{mag}\right)$ - our current data cannot distinguish between these two possibilities. In a histogram of the IR color index $J-K_{S}$, the two peaks of the bimodal distribution can be understood as single stars (blue peak at $J-K_{S}=-0.158$ ) and composite systems (red peak at $J-K_{S}=+0.311$ ). This bimodal distribution is also present in the approximately volume limited sample, again with the two peaks at $J-K_{S}=-0.154$ and +0.276 . Making a cut at $J-K_{S}=+0.05$, we found the ratio between single and composite sdB stars is 58:42 (favoring singles) in the magnitude limited sample, while in the volume limited sample it is 75:25 (favoring singles). Note that these ratios do not include any extrapolation to companions that do not affect the photometry (i.e., WD or late M dwarfs), since such extrapolation depends on the assumptions made about the absolute magnitude of the subdwarfs or their companions. The $J-K_{S}$ distribution for both the magnitude and volume samples can be described as the sum of two Gaussians with very little overlap and with fractions of single vs. composite in agreement with a sharp cut at $J-K_{S}=+0.05$. An interpretation of these ratios is difficult due to biases in the initial subdwarf catalog.

Comparison to other surveys of composite systems is difficult due to the unknown selection effects involved. Other surveys have generally concluded that $\gtrsim 50 \%$ of hot subdwarfs have late type companions (e.g., Allard et al. 1994; Bixler et al. 1991; Ferguson et al. 1984). However, these percentages depend greatly on the corrections made to account for late type companions that were undetectable by the survey (i.e., M-dwarfs) and the assumed luminosity of the subdwarfs or 
observed companions (i.e., main sequence or subgiant). Based on their observations of composite spectrum sdB stars from the PG survey (Green et al. 1986), Ferguson et al. (1984) extrapolated that about $50 \%$ of the sdB stars in the PG catalog had main sequence companions of G8 or later (and a few had companions earlier than G8). Allard et al. (1994) identified 31 composite candidates out of $100 \mathrm{sdB}$ stars observed photometrically, and deduced a fraction of sdB stars with cool main sequence companions of 54-66\%, when allowance was made for perceived selection effects. From intermediate resolution spectroscopy of the Ca II IR triplet region of $40 \mathrm{sdB}$ stars, and an assumed "typical" luminosity of a sdB star, Jeffery \& Pollacco (1998) claim that the seven composite systems they observed contained subgiant companions. Therefore they conclude that the the binary fraction must be much higher than what Allard et al. (1994) concluded based on assuming main sequence companions. From a sample of 40 UV selected hot subdwarfs, Bixler et al. (1991) found that eight showed composite spectra, and calculated that $65-100 \%$ of hot subdwarfs had a late type main sequence companion. However, our volume limited sample shows significantly fewer composites then our magnitude limited sample. Thus, by studying only magnitude limited samples (or even less well-defined samples) of hot subdwarfs, it is conceivable that the total number of composite systems could have been significantly over-estimated by these previous surveys (all other factors being equal).

The complete 2MASS catalog was released as this paper was nearing completion. We will address the full sky sample in a future contribution.

The authors thank E. M. Green for providing a list of spectroscopically observed hot subdwarfs, G. B. Berriman for his help interpreting the 2MASS catalog, and R. F. Green for helpful correspondence. RW thanks STScI for its hospitality.

This research has been supported in part by: NASA Grid Stars grant NAG5-9586, NASA GSRP grant NGT5-50399, and a NASA Space Grant Fellowship through the Pennsylvania Space Grant Consortium.

This publication makes use of: data products from the Two Micron All Sky Survey, which is a joint project of the University of Massachusetts and the Infrared Processing and Analysis Center/California Institute of Technology, funded by the National Aeronautics and Space Administration and the National Science Foundation; the USNOFS Image and Catalogue Archive operated by the United States Naval Observatory, Flagstaff Station (http://www.nofs.navy.mil/data/fchpix/); the SIMBAD database, operated at CDS, Strasbourg, France; and the Digitized Sky Surveys. The Digitized Sky Surveys were produced at the Space Telescope Science Institute under U.S. Government grant NAG W-2166. The images of these surveys are based on photographic data obtained using the Oschin Schmidt Telescope on Palomar Mountain and the UK Schmidt Telescope. The plates were processed into the present compressed digital form with the permission of these institutions. 


\section{REFERENCES}

Allard, F., Wesemael, F., Fontaine, G., Bergeron, P., \& Lamontagne, R. 1994, AJ, 107, 1565

Allard, F., Wesemael, F., Lamontagne, R., Bergeron, P., \& Fontaine, G. 1987, in IAU, Colloquium no. 95, The Second Conference on Faint Blue Stars, ed. A. G. D. Philip, D. S. Hayes, \& J. W. Liebert (Schenectady, NY: L. Davis Press Inc.), 611

Baade, W. 1944, ApJ, 100, 137

Beers, T. C., Preston, G. W., Shectman, S. A., Doinidis, S. P., \& Griffin, K. E. 1992, AJ, 103, 267

Berg, C., Wegner, G., Foltz, C. B., Chaffee, F. H., Jr., \& Hewett, P. C. 1992, ApJS, 78, 409

Bixler, J. V., Bowyer, S., \& Laget, M. 1991, A\&A, 250, 370

Brown, T. M., Ferguson, H. C., Davidsen, A. F., \& Dorman, B. 1997, ApJ, 482, 685

Brown, T. M., Bowers, C. W., Kimble, R. A., Sweigart, A. V., \& Ferguson, H. C. 2000, ApJ, 532, 308

Caloi, V. 1972, A\&A, 20, 357

Drilling, J. S., \& Landolt, A. U. 2000, Allen's Astrophysical Quantities, 4th Edition, ed. A. Cox (New York: Springer-Verlag), 381

de Boer, K. S., Drilling, J., Jeffery, C. S., \& Sion, E. M. 1997, in The Third Conference on Faint Blue Stars, ed. A. G. D. Philip, J. W. Liebert, \& R. A. Saffer (Schenectady, NY: L. Davis Press Inc.), 515

Drechsel, H., Heber, U., Napiwotzki, R., Østensen, R., Solheim, J.-E., Johannessen, F., Schuh, S. L., Deetjen, J., \& Zola, S. 2001, A\&A, 379, 893

Drilling, J. S., Moehler, S., Jeffery, C. S., Heber, U., \& Napiwotzki, R. 2000, in The Kth Reunion, ed. A. G. D. Philip (Schenectady, NY: L. Davis Press), 49

Drilling, J. S., Moehler, S., Jeffery, C. S., Heber, U., \& Napiwotzki, R. 2003, in Probing the Personalities of Stars and Galaxies, ed. A. G. D. Philip (Schenectady, NY: L. Davis Press), in press

Dworetsky, M. M., Lanning, H. H., Etzel, P. B., \& Patenaude, D. J. 1977, IBVS 1284

ESA 1997, The Hipparcos \& Tycho Catalogues, ESA SP-1200

Etzel, P. B., Lanning, H. H., Patenaude, D. J., \& Dworetsky, M. M. 1977, PASP, 89, 616

Ferguson, D. H., Green, R. F., \& Liebert, J. 1984, ApJ, 287, 320

Green, E. M., et al. 2003, ApJ, 583, 31 
Green, R. F., Schmidt, M., \& Liebert, J. 1986, ApJS, 61, 305

Greenstein, J. L., \& Sargent, A. I. 1974, ApJS 28, 157

Greggio, L., \& Renzini, A. 1990, ApJ, 364, 35

Heber, U. 1986, A\&A, 155, 33

Heber, U., Jordan, S., \& Weidemann, V. 1991, in White Dwarfs, Proceedings of the 7th European Workshop, ed. G. Vauclair \& E. Sion (Norwell, MA: Kluwer Academic Publishers), 109

Heber, U., Moehler, S., Napiwotzki, R., Thejll, P., \& Green, E. M. 2002, A\&A, 383, 938

Hewett, P. C., Foltz, C. B., \& Chaffee, F. H. 1995, AJ, 109, 1498

Høg, E., et al. 1998, A\&A, 335, L65

Høg, E., et al. 2000, A\&A, 355, L27

Humason, M. L., \& Zwicky, F. 1947, ApJ, 105, 85

Jeffery, C. S., \& Pollacco, D. L. 1998, MNRAS, 298, 179

Jeffery, C. S., Drilling, J. S., Harrison, P. M., Heber, U., Moehler, S. 1997 A\&AS, 125, 501

Johnson, H. L 1966, ARA\&A, 4, 193

Kilkenny, D., Heber, U., \& Drilling, J. S. 1988, SAAOC, 12, 1; and expanded electronic version 1992, D. Kilkenny private communication (KHD)

Kilkenny, D., Koen, C., O’Donoghue, D., \& Stobie, R. S. 1997, MNRAS, 285, 640

Kilkenny, D., O’Donoghue, D., Koen, C., \& van Wyk, F. 1998, MNRAS, 296, 329

Landolt, A. U. 1992, AJ, 104, 340

Liebert, J., Saffer, R. A., \& Green, E. M. 1994, AJ, 107, 1408

Maxted, P. F. L., Heber, U., Marsh, T. R., \& North, R. C. 2001, MNRAS, 326, 1391

Maxted, P. F. L., Marsh, T. R., Heber, U., Morales-Rueda, L., North, R. C., \& Lawson, W. A. 2002, MNRAS, 333, 231

Mengel, J. G., Norris, J., \& Gross, P. G. 1976, ApJ, 204, 488

Menzies, J. W., \& Marang, F. 1986, IAUS, 118, 305

Moehler, S., Heber, U., \& de Boer, K. S. 1990, A\&A, 239, 265

Monet, D., et al. 1996, USNO-SA1.0, (U.S. Naval Observatory, Washington DC) 
Mooney, C. J., Rolleston, W. R. J., Keenan, F. P., Pinfield, D. J., Pollacco, D. L., Dufton, P. L., \& Katsiyannis, A. C. 2000, A\&A, 357, 553

O'Connell, R. W. 1999, ARA\&A, 37, 603

O’Donoghue, D., Koen, C., Kilkenny, D., Stobie, R. S., \& Lynas-Gray, A. E. 1999, in ASP Conf. Ser. 169, 11th European Workshop on White Dwarfs, ed. J.-E. Solheim, \& E. G. Meištas (San Francisco: ASP), 149

Orosz, J. A., Wade, R. A., \& Harlow, J. J. B. 1997, AJ, 114, 317

Østensen, R., Heber, U., Silvotti, R., Solheim, J. E., Dreizler, S., \& Edelmann, H. 2001, A\&A, 378, 466

Perryman, M. A. C., et al. 1997, A\&A, 323, L49

Reid, N., Wegner, G., Wickramasinghe, D. T., Bessell, M. S. 1988, AJ, 96, 275

Saffer, R. A., Bergeron, P., Koester, D., \& Liebert, J. 1994, ApJ, 432, 351

Saffer, R. A., Green, E. M., \& Bowers, T. P. 2000, in ASP Conf. Ser. 226, 12th European Conference on White Dwarfs, ed. J. L. Provencal, H. L. Shipman, J. MacDonald, \& S. Goodchild (San Francisco: ASP), 408

Theissen, A., Moehler, S., Heber, U., Schmidt, J. H. K., \& de Boer, K. S. 1995, A\&A, 298, 577

Theissen, A., Moehler, S., \& Schmidt, J. H. 1992, in Lecture Notes in Physics no. 401, The Atmospheres of Early-Type Stars, ed. U. Heber, \& C. S. Jeffery (New York: Springer-Verlag), 264

Thejll, P., Flynn, C., Williamson, R., \& Saffer, R. 1997, A\&A, 317, 689

Thejll, P., Theissen, A., \& Jimenez, R. 1994, A\&A, 292, 457

Thejll, P., Ulla, A., \& MacDonald, J. 1995, A\&A, 303, 773

Turner, D. C. 1990, PASP, 102, 1331

Ulla, A., \& Thejll, P. 1998, A\&AS, 132, 1

Ulla, A., Zapatero Osorio, M. R., Pérez Hernández, F., \& MacDonald, J. 2001, A\&A, 369, 986

Viton, M., Deleuil, M., Tobin, W., Prevot, L., \& Bouchet, P. 1991, A\&A, 242, 175

Wesemael, F., Fontaine, G., Bergeron, P., Lamontagne, R., \& Green, R. F. 1992, AJ, 104, 203

Williams, T., McGraw, J. T., \& Grashuis, R. 2001, PASP, 113, 490 


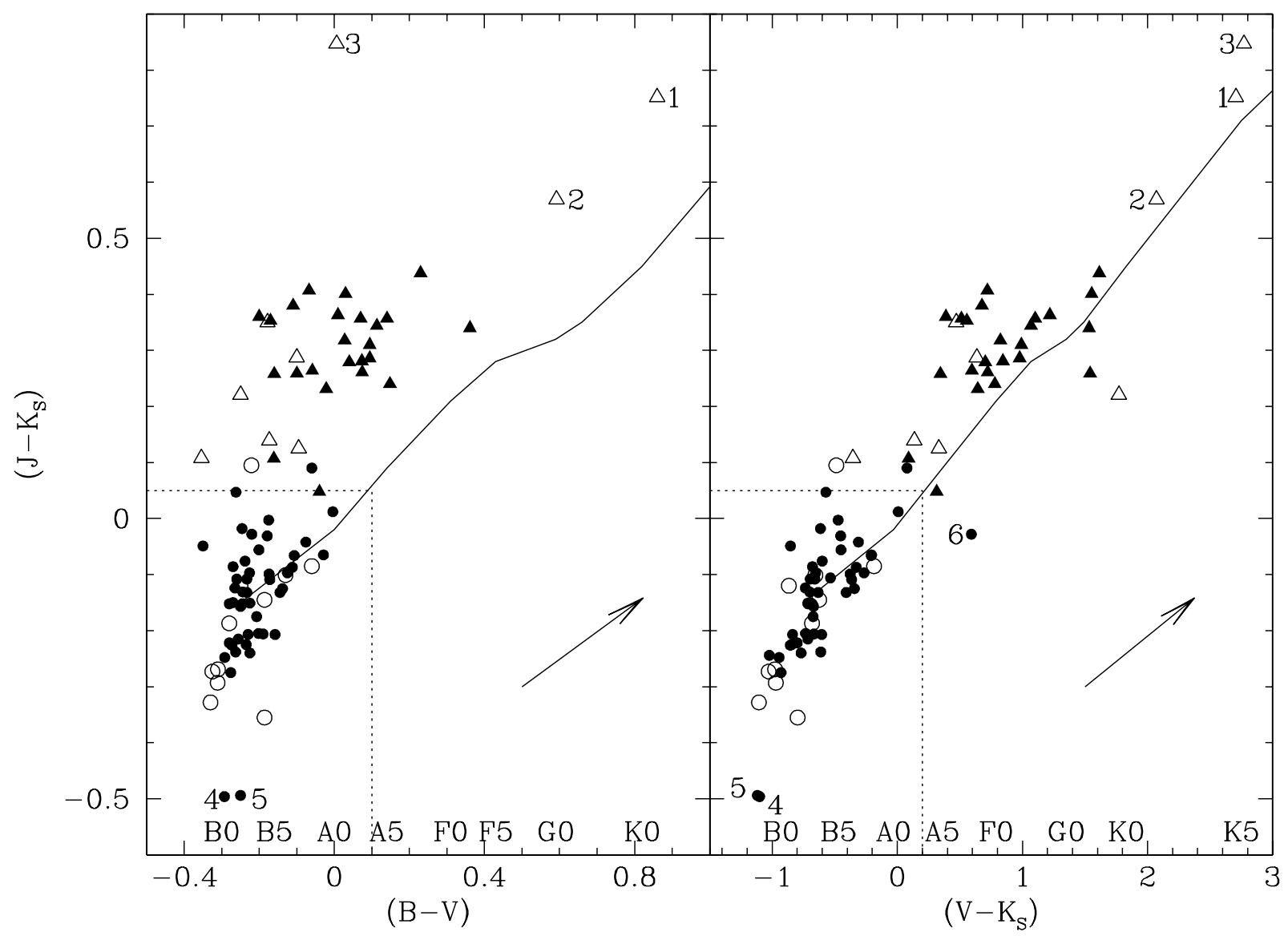

Fig. 1. - Color-color diagrams for reported single (circles) and composite (triangles) hot subdwarfs. Filled points represent hot subdwarfs in the sdB group (48 single, 24 composite), open points represent stars in the sdO group (10 single, 8 composite). The left panel shows $\left(J-K_{S}\right)$ vs. $(B-V)$, while the right panel shows $\left(J-K_{S}\right)$ vs. $\left(V-K_{S}\right)$. The diagonal solid line in each panel indicates the location of the Population I main sequence (with the approximate Pop I spectral types indicated along the $B-V$ and $V-K_{S}$ axes). The single hot subdwarfs are mostly contained within the box defined by: $B-V \lesssim+0.1, V-K_{S} \lesssim+0.2$, and $J-K_{S} \lesssim+0.05$, which is shown in each panel with a dotted line. Objects labeled 1-5 in both panels, and 6 in the right panel, are discussed further in the text (§3.1). The arrows indicate color shift due to one magnitude of extinction at $V$. 


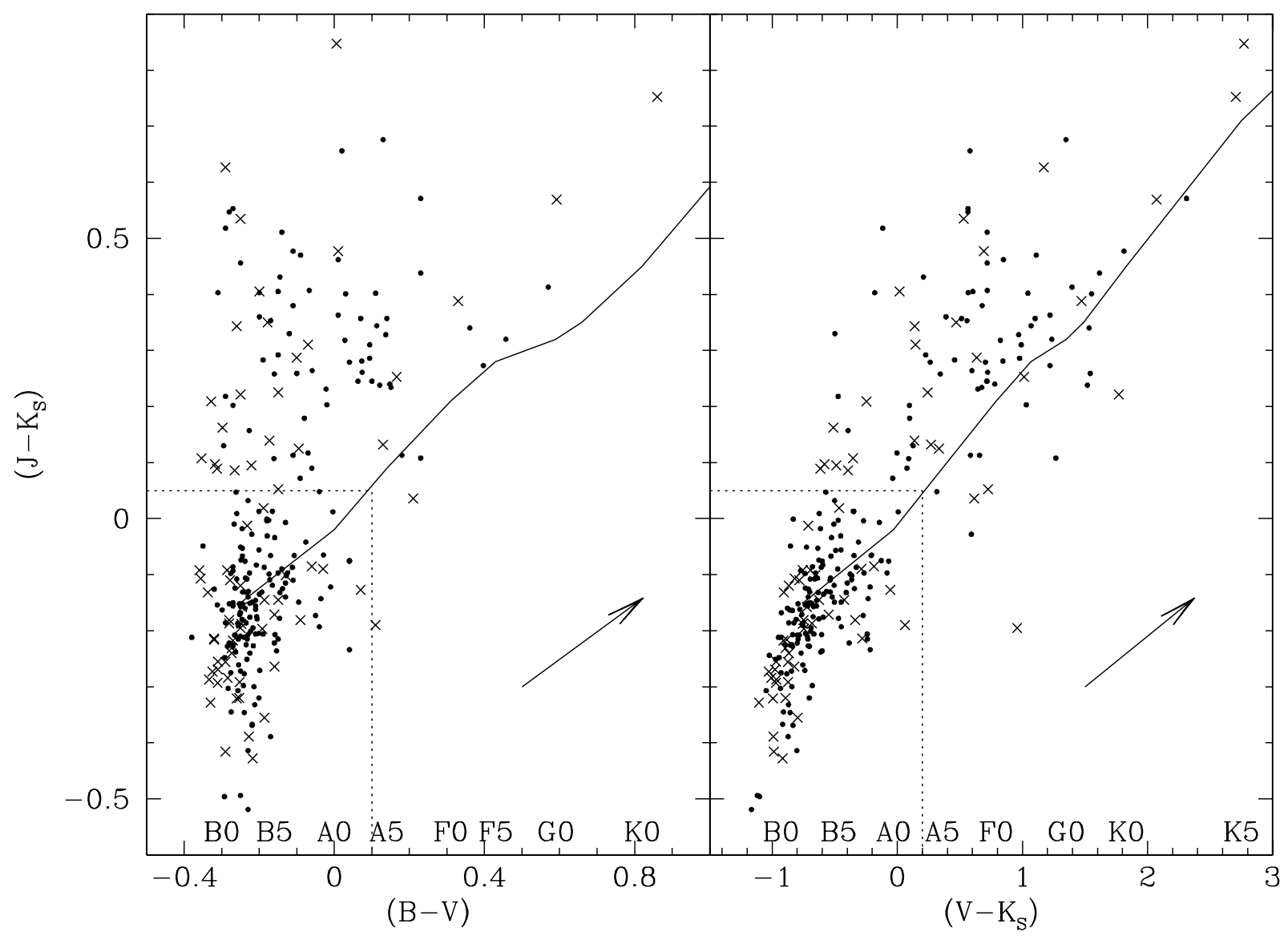

Fig. 2.- Same as Figure 1, except all hot subdwarfs with 2MASS 2IDR and visual photometry are shown. SdBs are denoted with circles and sdOs with crosses ("X"). There are 201 sdBs and $71 \mathrm{sdOs}$ in the left panel and $206 \mathrm{sdBs}$ and $71 \mathrm{sdOs}$ in the right; these totals include the reported composite and single stars of Figure 1 . One sdB, \#462 TON 56, has a published $B-V=-0.90$ which places it off the left side of the left panel, this $B V$ photometry from Green et al. (1986) is listed as uncertain; however, it is shown in the right panel $\left(V-K_{S}=+0.263, J-K_{S}=+0.279\right)$. Four other sdBs are missing from the left panel (but not the right), because they have no $B$ (or equivalent) measurements reported. 


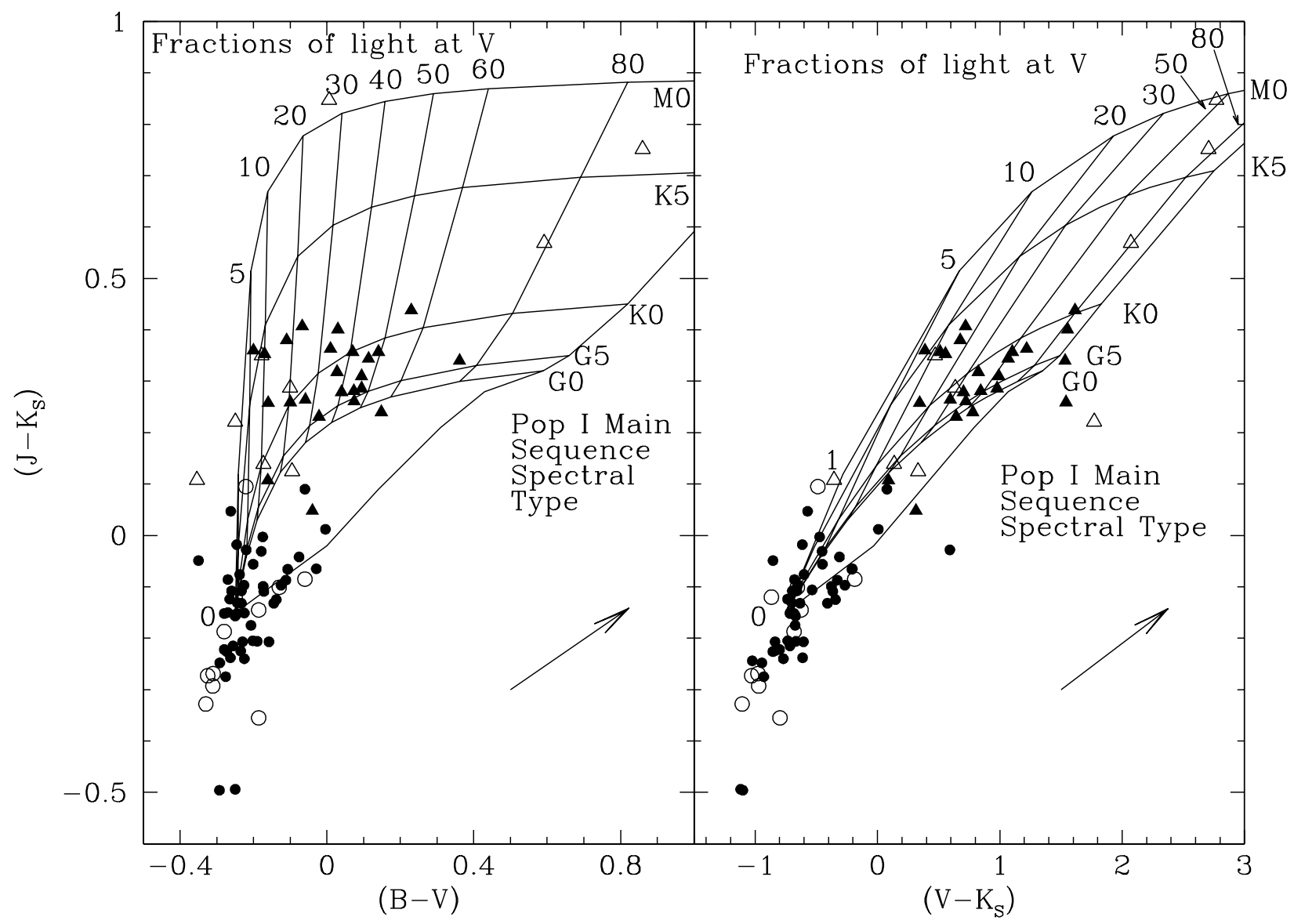

Fig. 3. - Grid of composite colors computed by combining the light from a hot subdwarf $(B-V \approx$ $\left.-0.25, V-K_{S} \approx-0.75, J-K_{S} \approx-0.15\right)$ with that of "typical" Pop I main sequence stars (G0, G5, K0, K5, and M0; Johnson 1966) assuming varying fractional contributions to the total light in the $V$ band by the late type star (fractions contributed by the companion: $0,1,5,10,20,30$, 40, 50, 60, 80, and 100\%). Circles are the reported single subdwarfs (solid: sdB, open: sdO) and triangles are the reported composite subdwarfs (solid: sdB, open: sdO) from Figure 1. The large arrows indicate the color change due to one magnitude of extinction at $V$. 


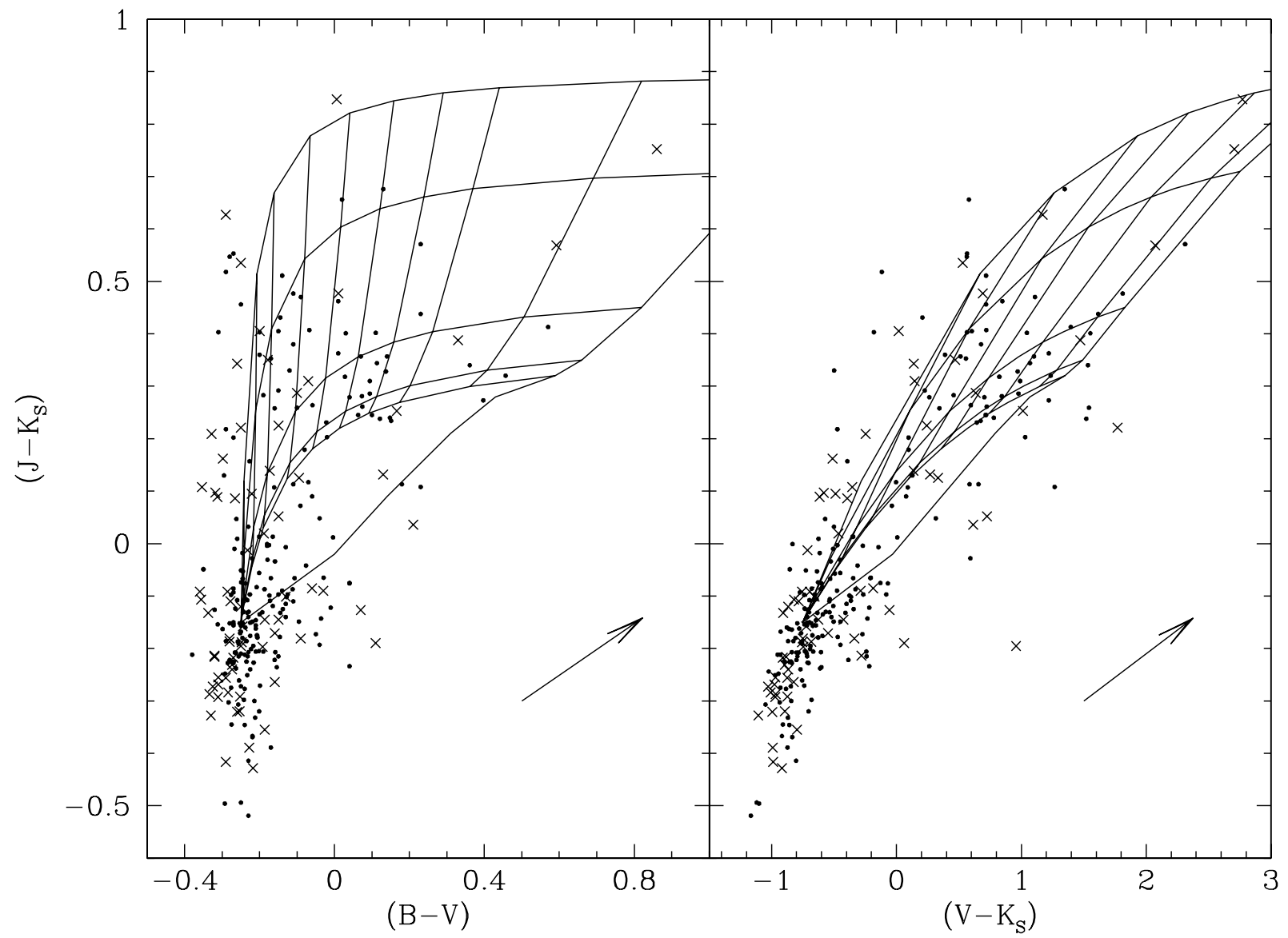

Fig. 4. - Same as Figure 3, except all sdBs (circles) and sdOs $(\times)$ with 2MASS 2IDR photometry are shown, as in Figure 2. 


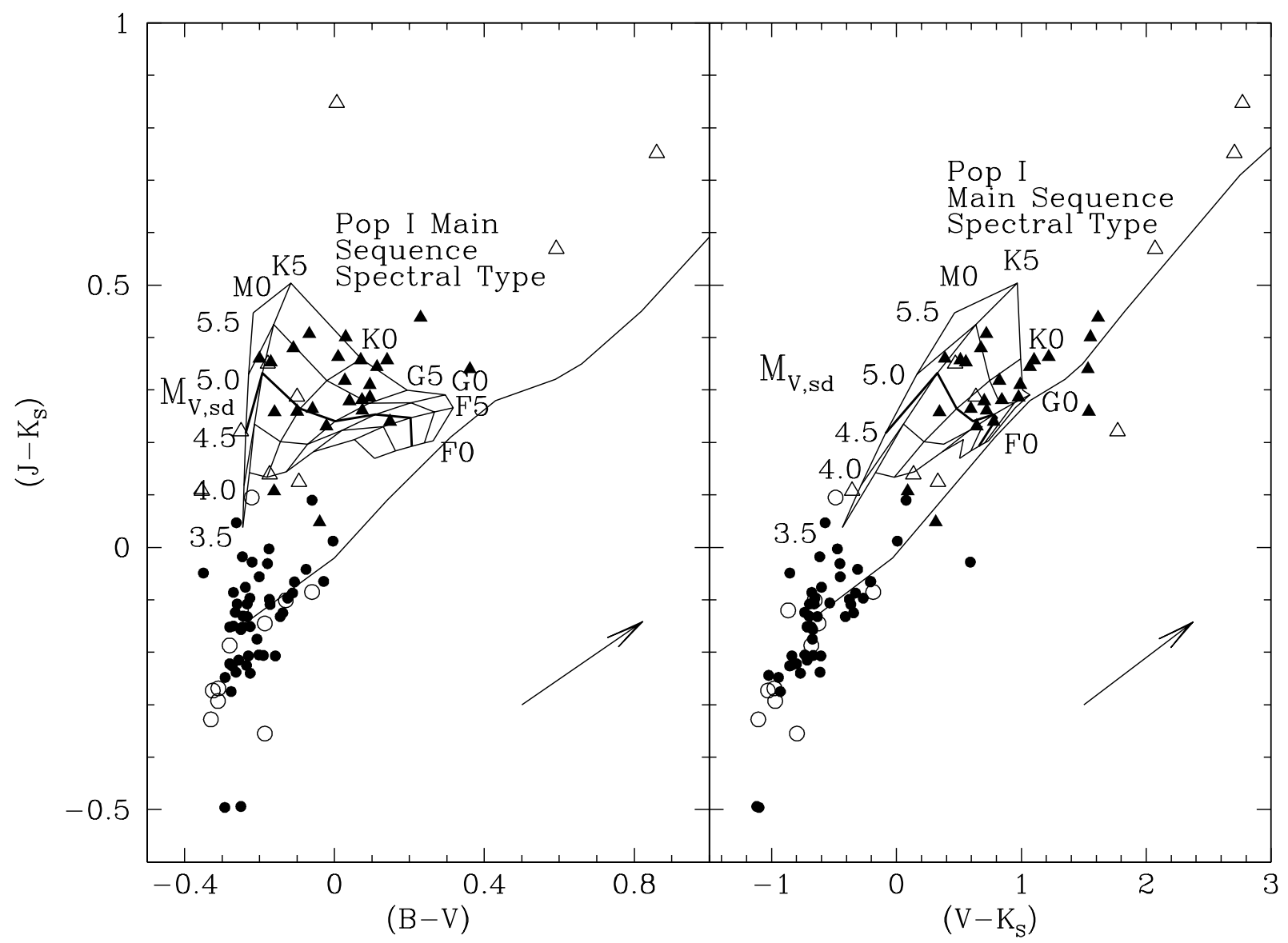

Fig. 5.- Grid of composite colors computed by combining the light from a hot subdwarf $(B-V \approx$ $\left.-0.25, V-K_{S} \approx-0.75, J-K_{S} \approx-0.15\right)$ with that of "typical" Pop I main sequence stars (F0, F5, G0, G5, K0, K5, and M0; Johnson 1966) and assuming values of $M_{V}$ for the subdwarf $\left(M_{V, \mathrm{sdB}}=3.5\right.$, 4.0, 4.5, 5.0, and 5.5) - the line for $M_{V, \mathrm{sdB}}=4.5$ has been darkened to help guide the eye. The lines for G5 and F5 have been left off the right plot for clarity. Circles are the reported single subdwarfs (solid: sdB, open: sdO) and triangles are the reported composite subdwarfs (solid: sdB, open: sdO) from Figure 1. The large arrows indicate the color change due to one magnitude of reddening. 


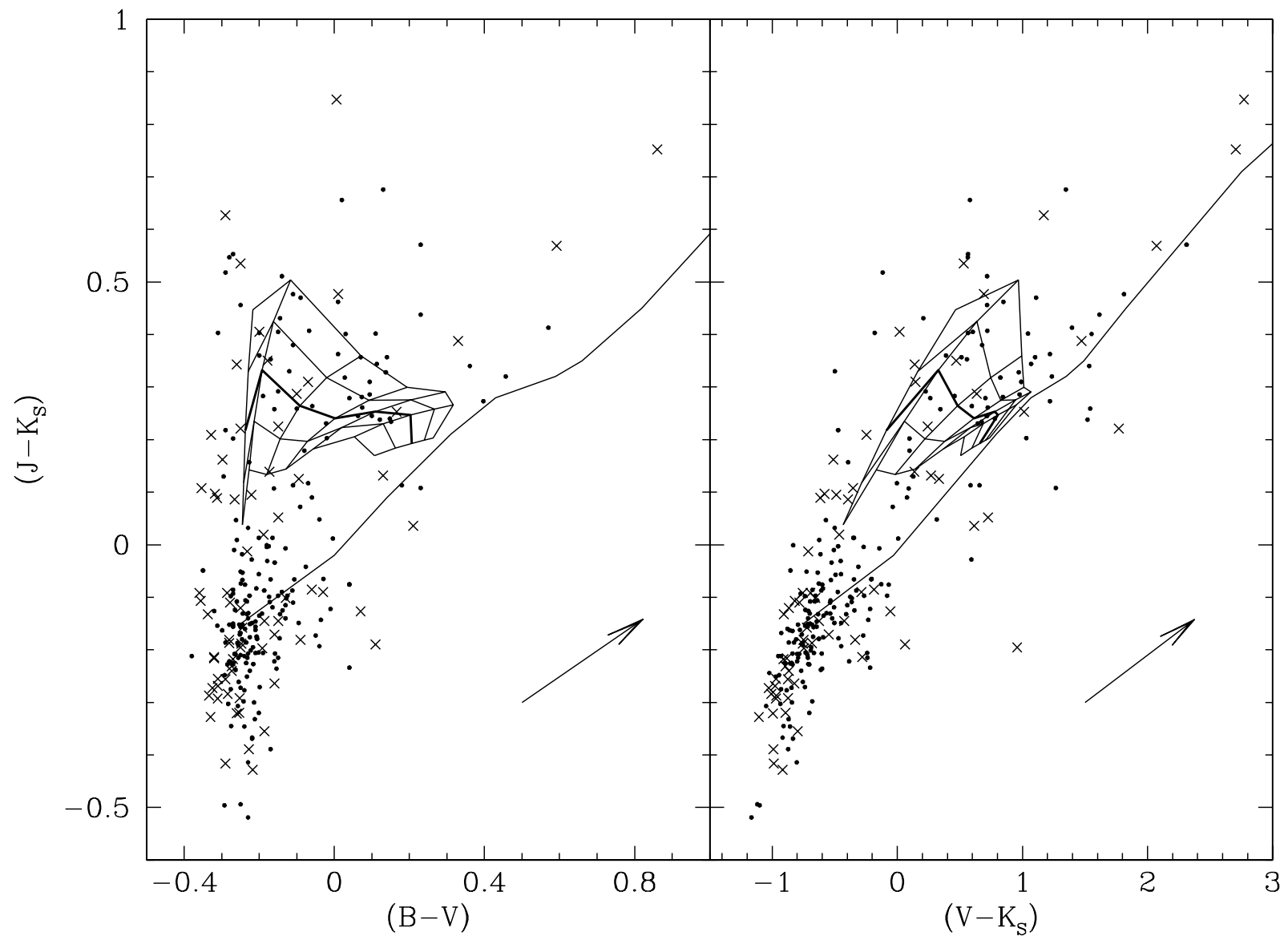

Fig. 6.- Same as Figure 5, except all sdBs (circles) and sdOs $(\times)$ with 2MASS 2IDR photometry are shown as in Figure 2. 


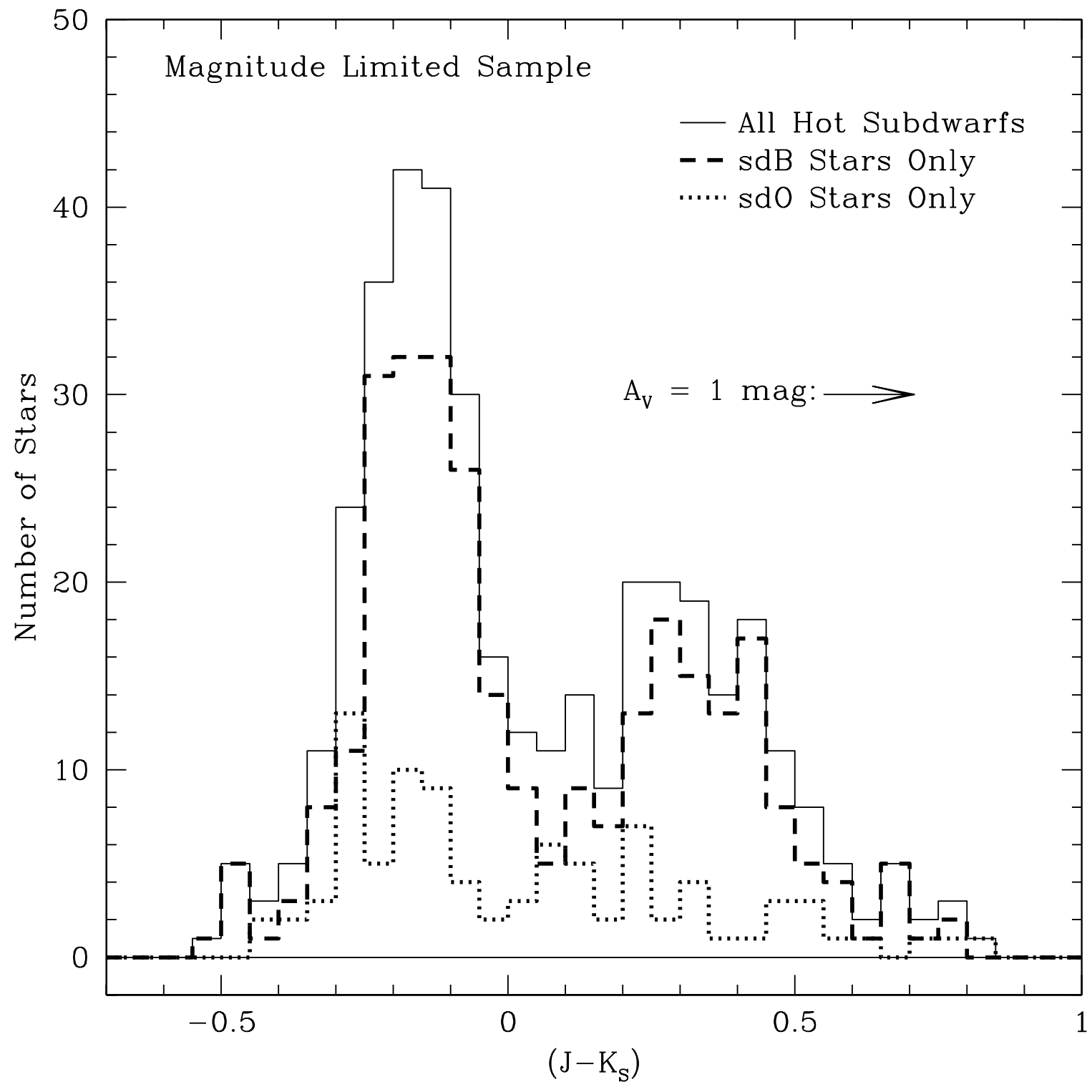

Fig. 7.- Histogram of $J-K_{S}$ for all hot subdwarf stars found in the 2MASS 2IDR (bin size $0.05 \mathrm{mag})$. The solid line indicates the full sample of hot subdwarfs, the dashed line indicates the sdB group, and the dotted line indicates the sdO group (these groups were defined in $\S 2$ ). Single subdwarfs are believed to reside in the left peak of the distribution centered around $J-K_{S} \approx-0.15$, while composite systems (sd+later type) reside in the right peak centered around $J-K_{S} \approx+0.3$, with the sdOs falling to slightly bluer colors than the sdBs in general. A tabulation of the numbers and fractions of single and composite hot subdwarfs can be found in Table 4. The arrow indicates the shift in $J-K_{S}$ color due to one magnitude of extinction at $V$. 


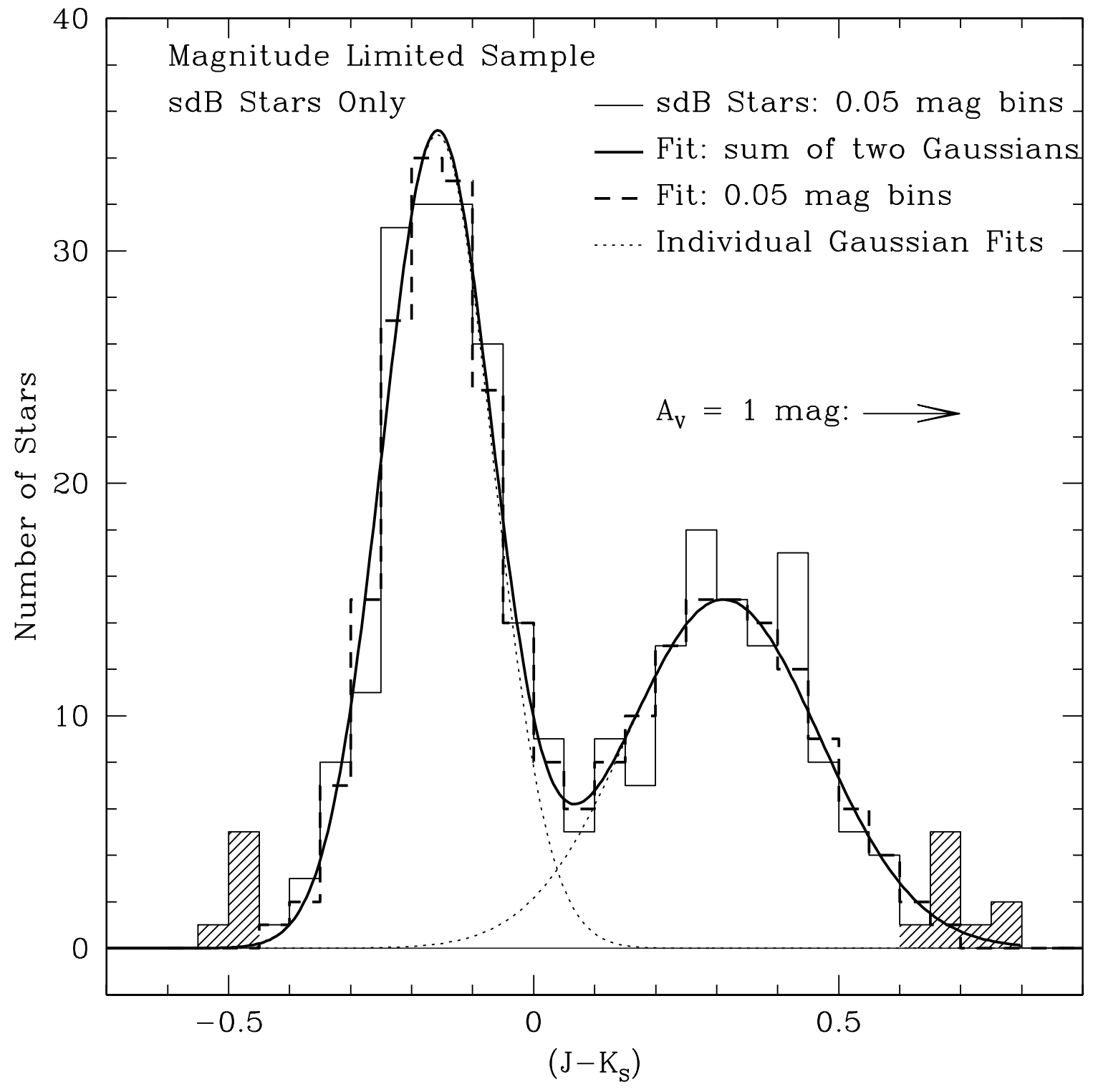

Fig. 8. - The sdB only distribution in $J-K_{S}$ (from Figure 7) fitted by the sum of two Gaussians. Shaded bins were excluded when calculating $\chi^{2}$ and $\chi_{\mathrm{R}}^{2}$. See $\S 3.4$ for further discussion, and Table 5 for a tabulation of the fit parameters. 


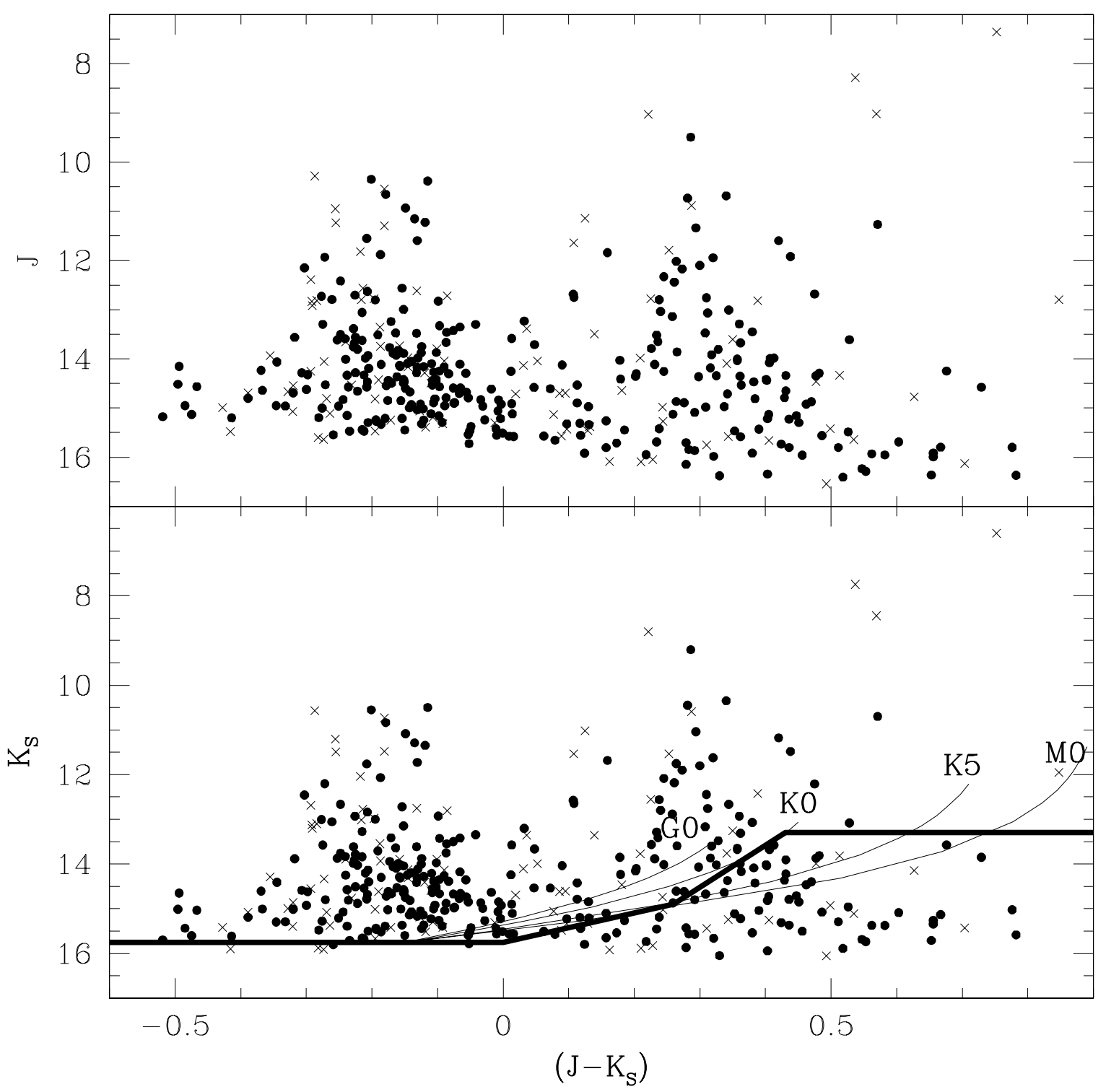

Fig. 9. - Color-magnitude diagrams of $J$ (top) and $K_{S}$ (bottom) vs. $\left(J-K_{S}\right)$. The sdBs are plotted as circles and the sdOs as crosses ( $\times$ "). The $K_{S}$ vs. $\left(J-K_{S}\right)$ diagram shows the cut made to get an approximately volume limited sample (thick line), as well as the limits (thin lines) for companions with colors corresponding to the Pop I main sequence spectral types: G0, K0, K5, and M0 (these limits are the lines from Figure 3 transformed into color-magnitude space by assuming an apparent magnitude for the subdwarf of $\left.K_{S}=15.75\right)$. 


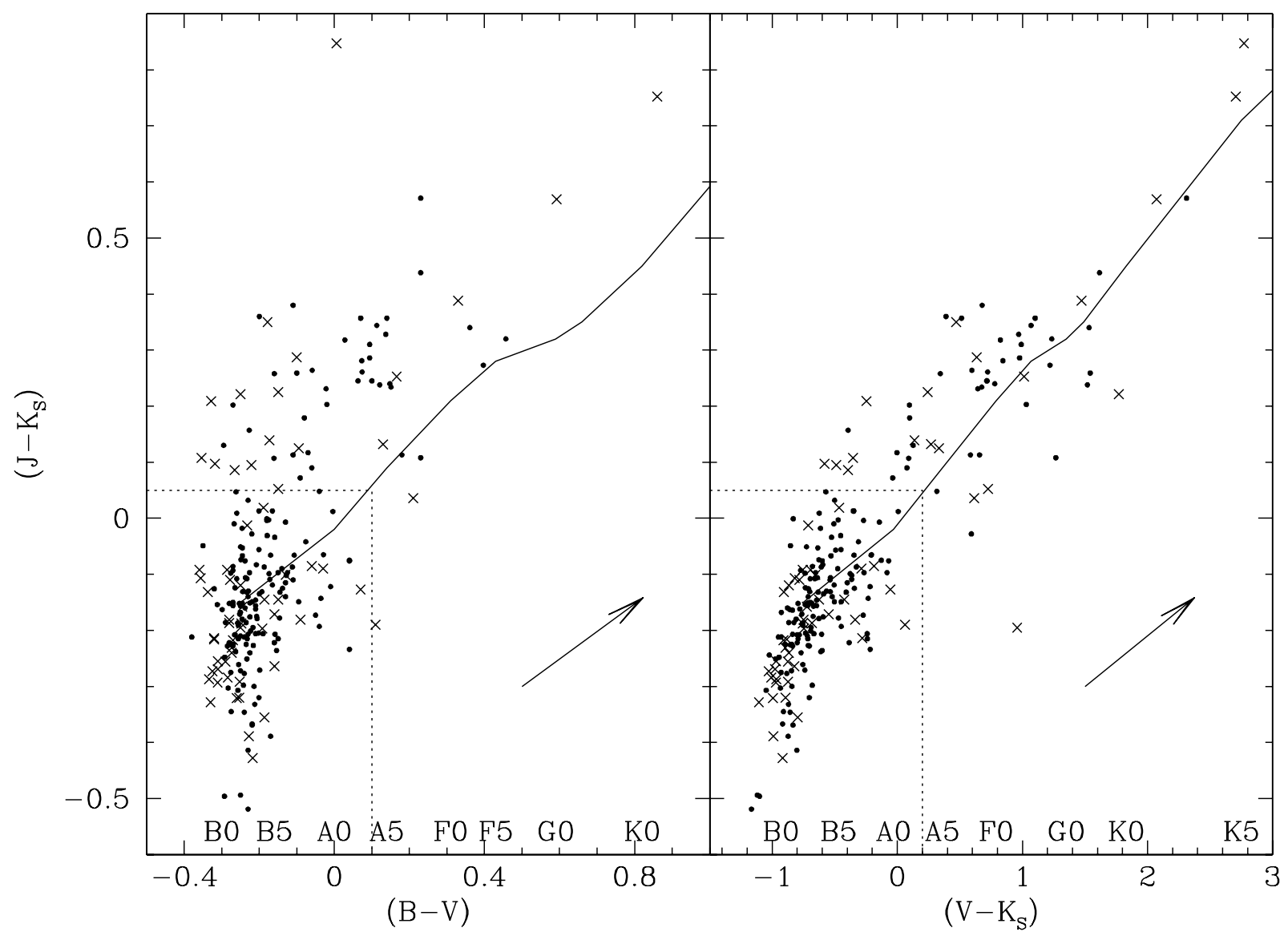

Fig. 10. - Same as Figure 2, except displaying only hot subdwarfs in the "volume limited" sample; sdBs (176 in the left panel, 180 in the right) are denoted with circles and sdOs (62 in both panels) with crosses ("X"). 


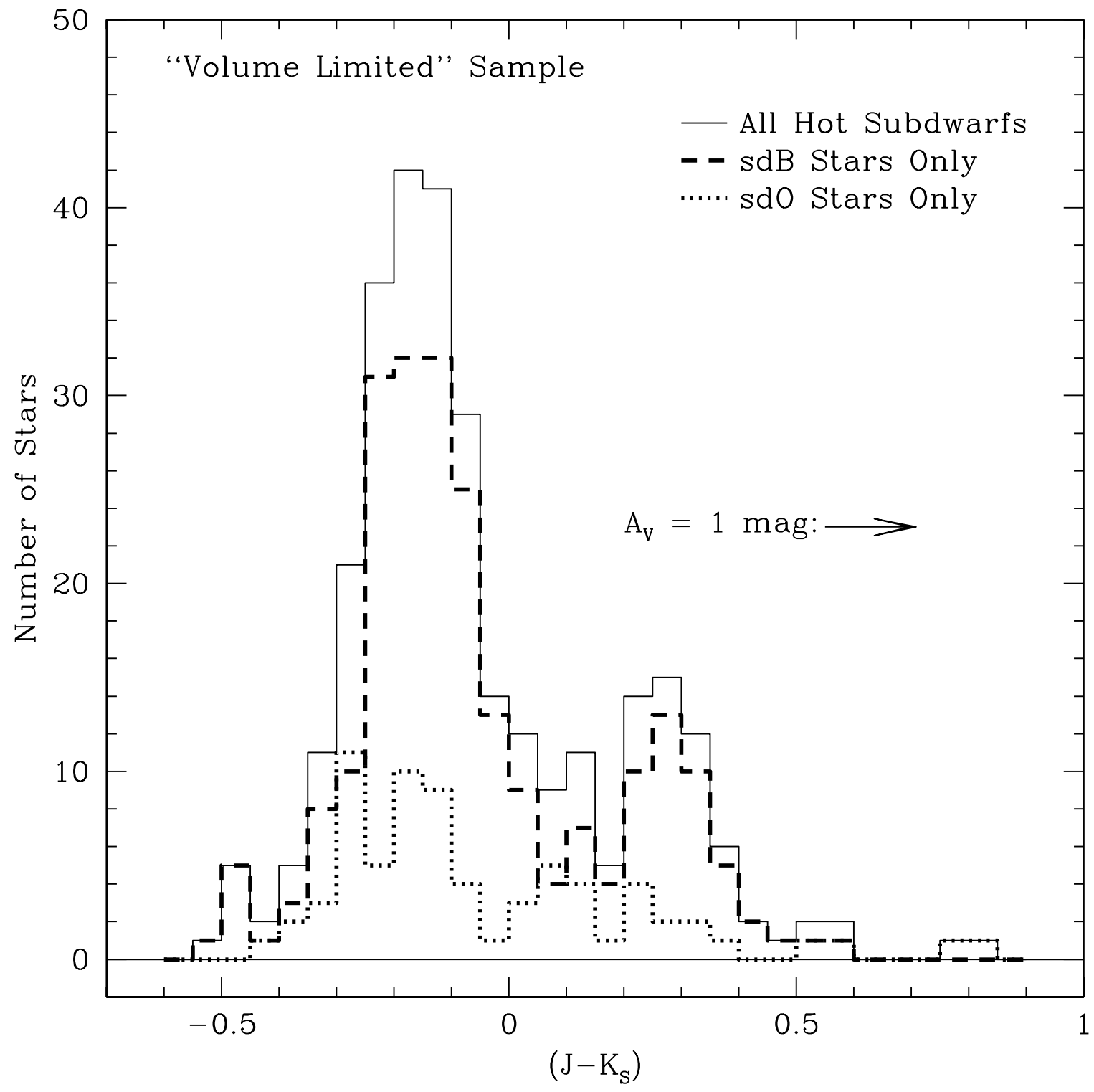

Fig. 11.- Same as Figure 7, except showing only hot subdwarfs in the "volume limited" sample. A tabulation of the numbers and fractions of single and composite hot subdwarfs can be found in Table 6 . The arrow indicates the shift in $J-K_{S}$ color due to one magnitude of extinction at $V$. 


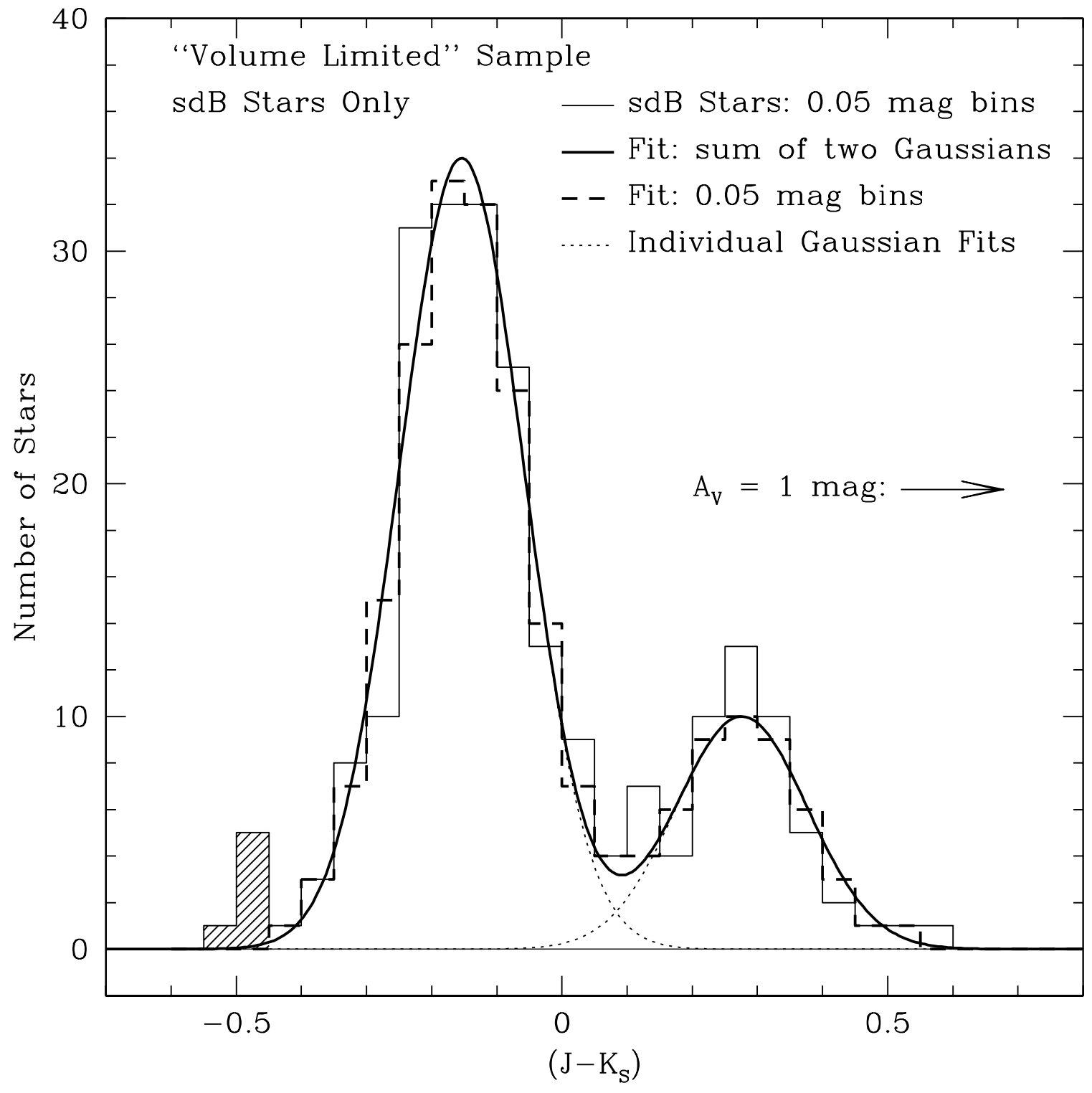

Fig. 12.- The "volume limited" sdB-only distribution in $J-K_{S}$ (from Figure 11), fitted by the sum of two Gaussians. Shaded bins were excluded when calculating $\chi^{2}$ and $\chi_{\mathrm{R}}^{2}$. See $\S 4.2$ for further discussion, and Table 7 for a tabulation of the fit parameters. 
Table 1. Updated Coordinates and Notes for All Subdwarfs with a 2MASS 2IDR Identification (ordered by RA).

\begin{tabular}{rllllll}
\hline \hline No. $^{1}$ & \multicolumn{1}{c}{ KHD ID $^{2}$} & RA (J2000) & Dec $(\mathrm{J} 2000)$ & Type & Notes $^{3}$ & SIMBAD ID $^{4}$ \\
\hline 1525 & CSHK 22957-23 & 000132.285 & -051916.76 & sdB & $\ldots$ & BPS CS 22957-23 \\
1526 & CSHK 22876-28 & 000137.625 & -353953.09 & sdB & $\ldots$ & BPS CS 22876-28 \\
1527 & PG 23595+1942 & 000208.520 & +195913.04 & sd & $\ldots$ & PG 2359+197 \\
1 & SB 7 & 000324.371 & -162106.34 & sdB & $\# 3($ CSHK 29517-41) & (BPS CS 29517-41) \\
6 & TON S 137 & 000430.992 & -242621.15 & sdO & $\# 5($ CSHK 29503-9) & (BPS CS 29503-9) \\
8 & CSHK 22876-35 & 000556.689 & -345306.49 & sdB & $\ldots$ & BPS CS 22876-35 \\
10 & TON S 140 & 000646.257 & -272053.60 & sdOB & $\# 11($ CSHK 29503-2) & (BPS CS 29503-2) \\
12 & PHL 678 & 000733.785 & +133558.70 & sdB & $\ldots$ & $\ldots$ \\
19 & TON S 144 & 001006.925 & -261256.94 & sdO & $\# 17$ (CSHK 29503-25) & (BPS CS 29503-25) \\
22 & PHL 2726 & 001227.751 & +035431.64 & sd (B) & $\ldots$ & $\ldots$ \\
\hline
\end{tabular}

Note. - The complete version of this table is in the electronic edition of the Journal. The printed edition contains only a sample.

${ }^{1}$ The sequence number, assigned by us, is not intended to be used as a new designator in the literature, but is useful in navigating the KHD catalog and cross-referencing this table with Table 2.

${ }^{2}$ The first identifier that appears in KHD for a given entry.

3 \#NNNN (Identifier) = indicates a duplicate entry sequence number and the first identifier listed for the duplicate in KHD (the duplicate entry is not otherwise listed in this table); $a=\# 87$, Heber et al. (2002) report that this pair is resolved into three components with HST: the sdO is the NW star of the "pair," while the SE "companion" star resolves into two components separated from the sdO by $3^{\prime \prime} 37$ and $4 . \prime 48 . \quad b=\# 284$, visual double, companion is M-type (KHD); $c=\# 293$, Ulla et al. (2001) report two close companions ("B" located $\leq 2$ ". $4 \mathrm{E}$, and "C" located $\sim 33^{\prime \prime} 5 \mathrm{NW}$ ); $d=\# 1337$ and 1322 were listed with the same name (CSHK 29495-21) by KHD when in fact they are two different objects: \#1322 is named correctly, while \#1337 is not - \#1337 should read CSHK 29495-63 in KHD.

${ }^{4}$ The identifiers listed in the last column are consistent with SIMBAD nomenclature. For example, the designator CSHK 22876-35 used by KHD for star \#8 in this table is equivalent to the SIMBAD designator BPS CS 22876-35, and the designator PG $23595+1942$ used by KHD for star \#1527 in this table is equivalent to the SIMBAD designator PG 2359+197. If no other ID is given in the last column, the identifier from KHD (given in column 2) is consistent with SIMBAD. In this way, one SIMBAD-compliant designator is available for cross-reference. The exceptions are the "HS" star \#390 HS 1000+4704, the "SC" star \#1165 SC 1721-3338 from Reid et al. (1988), and the "LBQS" stars from Berg et al. (1992, \#41,565, and 1405) which are not presently recognized by SIMBAD; although SIMBAD does use the LBQS designator for quasars from Hewett et al. (1995). In the case of a duplicate entry, if the duplicate's identifier listed in column 6 is not consistent with SIMBAD notation, then a SIMBAD-compliant name for the duplicate is given enclosed in parentheses. 
Table 2. Magnitudes and Colors for All Subdwarfs (ordered by J2000 RA).

\begin{tabular}{|c|c|c|c|c|c|c|c|c|c|c|c|c|c|}
\hline No. ${ }^{1}$ & $\mathrm{ID}^{2}$ & $J$ & $\sigma(J)^{3}$ & $H$ & $\sigma(H)^{3}$ & $K_{S}$ & $\sigma\left(K_{S}\right)^{3}$ & $J-K_{S}{ }^{3}$ & $\sigma\left(J-K_{S}\right)^{3}$ & $V(\text { or } y)^{4}$ & $B-V$ & Type & Notes $^{5}$ \\
\hline 1525 & CSHK22957-23 & 12.684 & 0.023 & 12.305 & 0.035 & 12.209 & 0.030 & +0.475 & 0.038 & $13.7 \mathrm{~B}$ & $\ldots$ & $\operatorname{sdB}$ & \\
\hline 1526 & CSHK22876-28 & 15.038 & 0.044 & 15.063 & 0.099 & 15.169 & 0.199 & -0.131 & 0.204 & $14.3 \mathrm{~B}$ & & $\operatorname{sdB}$ & \\
\hline 1527 & PG23595+1942 & 16.238 & 0.097 & 16.246 & 0.230 & 17.103 & & $<-0.865$ & & $15.44 \mathrm{P}$ & & sd & \\
\hline 1 & SB 7 & 12.686 & 0.032 & 12.631 & 0.036 & 12.579 & 0.034 & +0.107 & 0.047 & $12.67 y$ & -0.161 & $\mathrm{sdB}$ & $c$ \\
\hline 6 & TONS 137 & 14.534 & 0.037 & 14.714 & 0.064 & 14.765 & 0.113 & -0.231 & 0.119 & $13.87 y$ & -0.273 & $\mathrm{sdO}$ & \\
\hline 8 & CSHK22876-35 & 14.790 & 0.035 & 14.534 & 0.060 & 14.361 & 0.085 & +0.429 & 0.092 & $15.4 \mathrm{~B}$ & & $\mathrm{sdB}$ & \\
\hline 10 & TONS 140 & 14.543 & 0.031 & 14.687 & 0.065 & 14.863 & 0.101 & -0.320 & 0.106 & $13.97 y$ & -0.253 & $\mathrm{sdOB}$ & \\
\hline 12 & PHL 678 & 13.301 & 0.042 & 13.313 & 0.040 & 13.343 & 0.048 & -0.042 & 0.064 & 13.032 & -0.076 & $\mathrm{sdB}$ & $s, \mathrm{AEA}$ \\
\hline 19 & TONS 144 & 12.782 & 0.030 & 12.568 & 0.024 & 12.557 & 0.030 & +0.225 & 0.042 & $12.8 y$ & -0.15 & $\mathrm{sdO}$ & \\
\hline 22 & PHL 2726 & 13.329 & 0.030 & 13.397 & 0.030 & 13.426 & 0.046 & -0.097 & 0.055 & $13.16 y$ & -0.125 & sd (B) & $s$ \\
\hline
\end{tabular}

Note. - The complete version of this table is in the electronic edition of the Journal. The printed edition contains only a sample.

${ }^{1}$ The sequence number, assigned by us, is not intended to be used as a new designator in the literature, but is useful in navigating the KHD catalog and cross-referencing this table with Table 1.

${ }^{2}$ Identifiers listed as they appear in KHD. SIMBAD-compliant names are given in Table 1.

${ }^{3}$ Reported errors are $1 \sigma$. If no error is listed, then the star was not detected in that band, and the quoted magnitude is a $95 \%$ confidence upper limit, for $J-K_{S}$ the reported value is the maximum color index when an upper limit on $K_{S}$ is reported, and it is the minimum color index for an upper limit on $J$.

${ }^{4} y=$ Strömgren $y$ magnitude listed instead of Johnson $V ; \mathrm{P}, \mathrm{J}$, or B $=$ Photographic magnitude listed for $V$; colon (:) = uncertain magnitude.

${ }^{5} s=$ reported single sdB or sdO star used to determine the single hot subdwarf locus (see $\S 3.1$ and Figure 1 ); $c=$ reported composite sdB or sdO star used to determine the composite hot subdwarf locus (see $\$ 3.1$ and Figure 1); $C C=2 \mathrm{MASS}$ magnitudes listed are for a nearby companion only (the hot subdwarf was not detected); $n=$ excluded from the sample because of a "non-subdwarf" classification; AEA $=V$ and $B-V$ from Allard et al. (1994); BBL $=$ \# $1076 \& 1095$ had synthetic measurements converted from Tycho magnitudes (see $\$ 2.3): V_{T}=10.194,\left(B_{T}-V_{T}\right)=+0.111$ (ESA 1997); WMG $=V$ and $B-V$ from Williams et al. (2001); $a=\# 87$, Heber et al. (2002) report this pair is resolved into three components with HST: the sdO is the NW star of the "pair," while the SE "companion" star resolves into two components separated from the sdO by $3^{\prime \prime} 37$ and $4^{\prime \prime} 48 ; b=\# 203$, 2MASS noted a horizontal stripe due to bright star affecting the $H$ magnitude only, however, no stripe or nearby bright star could be seen in the 2MASS image; $d=\# 284$, visual double, companion is M-type (KHD) (only the companion was detected in the 2MASS 2IDR, thus it was excluded from the analysis); $e=\# 293$, Ulla et al. (2001) report two close companions ("B" located $\leq 2$ "'4 E, and "C" located $\sim 3$ " 5 NW); $f=\# 311$, Strömgren photometry is from Wesemael et al. (1992) but was not listed in KHD even though observations for other stars from Wesemael et al. (1992) were included in KHD; $g$ = \#428, IR excess possibly due to a wind (Thejll et al. 1995). 
Table 3. Breakdown of the Hot Subdwarf sample discussed in $\S 2$.

\begin{tabular}{|c|c|}
\hline Sub-Sample & Number of Objects \\
\hline \multicolumn{2}{|l|}{1527 Objects in KHD } \\
\hline Unrecovered & 57 \\
\hline Duplicate & 11 \\
\hline Input to 2MASS 2IDR & 1459 \\
\hline \multicolumn{2}{|c|}{593 IDs from 1459 input to 2MASS 2IDR } \\
\hline Subdwarfs & 571 \\
\hline Non-subdwarfs & 22 \\
\hline \multicolumn{2}{|c|}{571 Hot Subdwarfs from 2MASS 2IDR } \\
\hline With 2MASS $J$ and $K_{S}$ & 385 \\
\hline With 2MASS $J$ but $95 \%$ upper confidence on $K_{S}$ & 180 \\
\hline Detection of close companion to subdwarf only & 6 \\
\hline With $V($ or $y)$ & 348 \\
\hline With $B V$ (or uvby) & 339 \\
\hline \multicolumn{2}{|l|}{385 with $J$ and $K_{S}$} \\
\hline $\mathrm{sdB} / \mathrm{sd}$ & 294 \\
\hline $\mathrm{sdO} / \mathrm{sdOB}$ & 91 \\
\hline With $V($ or $y)$ & 277 \\
\hline With $B V$ (or uvby) & 273 \\
\hline \multicolumn{2}{|l|}{277 with $V$ (or $y), J$, and $K_{S}$} \\
\hline $\mathrm{sdB} / \mathrm{sd}$ & 206 \\
\hline $\mathrm{sdO} / \mathrm{sdOB}$ & 71 \\
\hline \multicolumn{2}{|l|}{273 with $B V$ (or uvby), $J$, and $K_{S}$} \\
\hline $\mathrm{sdB} / \mathrm{sd}$ & 202 \\
\hline $\mathrm{sdO} / \mathrm{sdOB}$ & 71 \\
\hline
\end{tabular}


Table 4. Breakdown of hot subdwarf colors based on $J-K_{S}$ for the entire sample discussed in $\S 3.3$.

\begin{tabular}{lccccc}
\hline \hline & \multicolumn{2}{c}{ Numbers } & \multicolumn{2}{c}{ Percentages $^{1}$} \\
Group & Total & Blue $^{2}$ & Red $^{3}$ & Blue $^{2}$ & Red $^{3}$ \\
\hline sdB & 294 & 171 & 123 & $58 \pm 6 \%$ & $42 \pm 6 \%$ \\
sdO & 91 & 48 & 43 & $53 \pm 10 \%$ & $47 \pm 10 \%$ \\
\hline Both & 385 & 223 & 162 & $58 \pm 5 \%$ & $42 \pm 5 \%$ \\
\hline
\end{tabular}

${ }^{1}$ Errors reported are $95 \%$ confidence sampling errors of a binomial distribution.

2 "Blue" is defined as $J-K_{S}<+0.05$ for the sdBs and the total sample, and $J-K_{S}<-0.05$ for sdOs.

3 "Red" is defined as $J-K_{S}>+0.05$ for the sdBs and the total sample, and $J-K_{S}>-0.05$ for sdOs. 
Table 5. Parameters for the Gaussian fit discussed in $\S 3.4$ and shown in Figure 8.

\begin{tabular}{lcc}
\hline \hline Parameter & Blue Gaussian & Red Gaussian $^{1}$ \\
\hline Center & $-0.158_{-0.005}^{+0.004}$ & $+0.311_{-0.004}^{+0.014}$ \\
Amplitude & $35_{-2}^{+3}$ & $15 \pm 2$ \\
Width & $0.091_{-0.002}^{+0.009}$ & $0.158_{-0.020}^{+0.008}$ \\
\hline
\end{tabular}

Integral of Best Fit: 278

\footnotetext{
${ }^{1}$ Errors reported are $95 \%$ marginal confidence intervals.
} 
Table 6. Breakdown of hot subdwarf colors based on $J-K_{S}$ for the "volume limited" sample discussed in $\S 4.2$.

\begin{tabular}{lccccc}
\hline \hline & \multicolumn{2}{c}{ Numbers } & \multicolumn{2}{c}{ Percentages $^{1}$} \\
Group & Total & Blue $^{2}$ & Red $^{3}$ & Blue $^{2}$ & Red $^{3}$ \\
\hline sdB & 228 & 170 & 58 & $75 \pm 6 \%$ & $25 \pm 6 \%$ \\
sdO & 72 & 45 & 27 & $62.5 \pm 11 \%$ & $37.5 \pm 11 \%$ \\
\hline Both & \multirow{2}{*}{300} & 219 & 81 & $73 \pm 5 \%$ & $27 \pm 5 \%$ \\
\hline
\end{tabular}

${ }^{1}$ Errors reported are $95 \%$ confidence sampling errors of a binomial distribution.

2 "Blue" is defined as $J-K_{S}<+0.05$ for the sdBs and the total sample, and $J-K_{S}<-0.05$ for sdOs.

3 "Red" is defined as $J-K_{S}>+0.05$ for the sdBs and the total sample, and $J-K_{S}>-0.05$ for sdOs. 
Table 7. Parameters for the Gaussian fit to the "volume limited" sample discussed in $\S 4.2$ and shown in Figure 12.

\begin{tabular}{lcc}
\hline \hline Parameter & Blue Gaussian $^{1}$ & Red Gaussian \\
\hline Center & $-0.154_{-0.006}^{+0.013}$ & $+0.276_{-0.006}^{+0.002}$ \\
Amplitude & $34 \pm 1$ & $10 \pm 1$ \\
Width & $0.096_{-0.004}^{+0.005}$ & $0.101_{-0.005}^{+0.002}$ \\
\hline
\end{tabular}

Integral of Best Fit: 214

${ }^{1}$ Errors reported are $95 \%$ marginal confidence intervals. 